\section{Crystal structure of di-n-butylbis $\left(\eta^{5}-\right.$ pentamethylcyclopentadienyl)- hafnium(IV)}

Perdita Arndt, ${ }^{\text {a }}$ Kathleen Schubert, ${ }^{\text {a }}$ Vladimir V. Burlakov, ${ }^{\mathrm{b}}$ Anke Spannenberg ${ }^{\mathrm{a}}$ and Uwe Rosenthal ${ }^{\mathrm{a} *}$

aLeibniz-Institut für Katalyse e. V. an der Universität Rostock, Albert-Einstein-Str. 29a, 18059 Rostock, Germany, and ${ }^{\mathbf{b}} \mathrm{A}$. N. Nesmeyanov Institute of Organoelement Compounds, Russian Academy of Sciences, Vavilov St 28, 119991, Moscow, Russian Federation. *Correspondence e-mail: uwe.rosenthal@catalysis.de

Received 9 December 2014; accepted 5 January 2015

Edited by A. V. Yatsenko, Moscow State University, Russia

The crystal structure of the title compound, $\left[\mathrm{Hf}\left(\mathrm{C}_{10} \mathrm{H}_{15}\right)_{2}\left(\mathrm{C}_{4} \mathrm{H}_{9}\right)_{2}\right]$, reveals two independent molecules in the asymmetric unit. The diffraction experiment was performed with a racemically twinned crystal showing a 0.529 (5):0.471 (5) component ratio. Each $\mathrm{Hf}^{\mathrm{IV}}$ atom is coordinated by two pentamethylcyclopentadienyl and two $n$ butyl ligands in a distorted tetrahedral geometry, with the cyclopentadienyl rings inclined to one another by 45.11 (15) and $45.37(16)^{\circ}$. In contrast to the isostructural di(n-butyl)bis$\left(\eta^{5}\right.$-pentamethylcyclopentadienyl)zirconium(IV) complex with a noticeable difference in the Zr-butyl bonding, the $\mathrm{Hf}-\mathrm{C}_{\text {butyl }}$ bond lengths differ from each other by no more than 0.039 (3) ̊.

Keywords: crystal structure; hafnocene; $n$-butyl; racemic twin.

CCDC reference: 1042126

\section{Related literature}

For the synthesis of the title compound, see: Schock \& Marks (1988), and for that of the corresponding unsubstituted cyclopentadienyl complex, see: Burlakov et al. (2008). For the use of these complexes as the starting materials for various reactions, see: Burlakov et al. (2009). For the structure of the isostructural zirconocene complex, see: Ernst et al. (2004), and of an ansa-zirconocene with an additional $\mathrm{Zr}-\mathrm{N}$ bond, see: Paolucci et al. (1997). For $\left[\mathrm{Mo}\left(\mathrm{C}_{5} \mathrm{H}_{5}\right)_{2}\left(\mathrm{C}_{4} \mathrm{H}_{9}\right)_{2}\right]$, see: Calhorda et al. (1991).

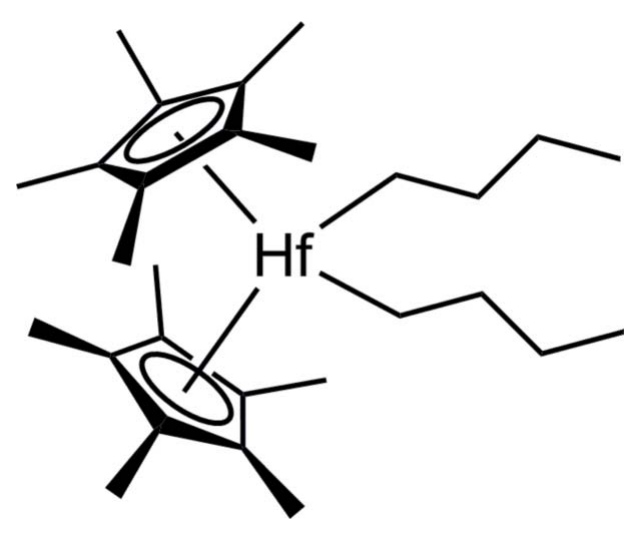

2. Experimental

\subsection{Crystal data \\ $\left[\mathrm{Hf}\left(\mathrm{C}_{10} \mathrm{H}_{15}\right)_{2}\left(\mathrm{C}_{4} \mathrm{H}_{9}\right)_{2}\right]$ \\ $M_{r}=563.15$ \\ Orthorhombic, $P 2_{1} 2_{1} 2_{1}$ \\ $a=17.1572(3) \AA$ \\ $b=17.2320(3) \AA$ \\ $c=17.2966(3) \AA$}

\subsection{Data collection \\ Bruker Kappa APEXII DUO CCD diffractometer \\ Absorption correction: multi-scan \\ (SADABS; Bruker, 2008) \\ $T_{\min }=0.30, T_{\max }=0.54$}

\subsection{Refinement}

$R\left[F^{2}>2 \sigma\left(F^{2}\right)\right]=0.018$

$w R\left(F^{2}\right)=0.038$

$S=1.06$

13236 reflections

548 parameters

$\mathrm{H}$-atom parameters constrained

$$
\begin{aligned}
& V=5113.79(15) \AA^{3} \\
& Z=8 \\
& \text { Mo } K \alpha \text { radiation } \\
& \mu=4.09 \mathrm{~mm}^{-1} \\
& T=170 \mathrm{~K} \\
& 0.39 \times 0.27 \times 0.17 \mathrm{~mm}
\end{aligned}
$$

123798 measured reflections 13236 independent reflections 12433 reflections with $I>2 \sigma(I)$ $R_{\text {int }}=0.037$
Data collection: APEX2 (Bruker, 2008); cell refinement: SAINT (Bruker, 2008); data reduction: $S A I N T$; program(s) used to solve structure: SHELXS97 (Sheldrick, 2008); program(s) used to refine structure: SHELXL2014 (Sheldrick, 2008); molecular graphics: XP in SHELXTL (Sheldrick, 2008); software used to prepare material for publication: SHELXL2014.

\section{Acknowledgements}

We would like to thank our technical and analytical staff for assistance. Financial support by Deutsche Forschungsgemeinschaft (RO 1269/9-1) and the Russian Foundation for Basic Research (project code 15-03-03485) is gratefully acknowledged.

Supporting information for this paper is available from the IUCr electronic archives (Reference: YK2105). 


\section{References}

Bruker (2008). APEX2, SAINT and SADABS. Bruker AXS Inc., Madison, Wisconsin, USA.

Burlakov, V. V., Beweries, T., Bogdanov, V. S., Arndt, P., Baumann, W., Petrovskii, P. V., Spannenberg, A., Lyssenko, K. A., Shur, V. B. \& Rosenthal, U. (2009). Organometallics, 28, 2864-2870.

Burlakov, V. V., Bogdanov, V. S., Lyssenko, K. A., Petrovskii, P. V., Beweries, T., Arndt, P., Rosenthal, U. \& Shur, V. B. (2008). Russ. Chem. Bull. 57, 13191320.
Calhorda, M. J., Carrondo, M. A. A. F. de C. T., Dias, A. R., Galvão, A. M., Garcia, M. H., Martins, A. M., Minas da Piedade, M. E., Pinheiro, C. I., Romão, C. C., Simões, J. A. M. \& Veiros, L. F. (1991). Organometallics, 10, 483-494.

Ernst, R. D., Harvey, B. G. \& Arif, A. M. (2004). Z. Kristallogr. New Cryst. Struct., 219, 398-400.

Flack, H. D. (1983). Acta Cryst. A39, 876-881.

Paolucci, G., Pojana, G., Zanon, J., Lucchini, V. \& Avtomonov, E. (1997). Organometallics, 16, 5312-5320.

Schock, L. E. \& Marks, T. J. (1988). J. Am. Chem. Soc. 110, 7701-7715.

Sheldrick, G. M. (2008). Acta Cryst. A64, 112-122. 


\section{supporting information}

Acta Cryst. (2015). E71, m19-m20 [doi:10.1107/S2056989015000092]

\section{Crystal structure of di-n-butylbis( $\eta^{5}$-pentamethylcyclopentadienyl)hafnium(IV)}

\section{Perdita Arndt, Kathleen Schubert, Vladimir V. Burlakov, Anke Spannenberg and Uwe Rosenthal}

\section{S1. Synthesis and crystallization}

The title compound was synthesized as described by Schock \& Marks (1988). To a suspension of $\mathrm{Cp}_{2}{ }_{2} \mathrm{HfCl}_{2}(1.00 \mathrm{~g}, 1.94$ $\mathrm{mmol})$ in $20 \mathrm{ml}$ of diethylether were added $3.0 \mathrm{ml}$ of $n-\mathrm{BuLi}(1.6 \mathrm{M}, 4.8 \mathrm{mmol})$ in diethylether. The solution was stirred for 3 days at RT before the ether was removed in vacuo. The residue was extracted with $n$-hexane three times, the collected filtrates were reduced in vacuo to $10 \mathrm{ml}$. At $-78^{\circ} \mathrm{C}$ colorless crystals were formed within two weeks $(0,545 \mathrm{~g}$, yield 50\%).

${ }^{1} \mathrm{H}$ NMR (300 MHz, $\left.\mathrm{C}_{6} \mathrm{D}_{6}\right): \delta=-0.14\left(\mathrm{t}, 4 \mathrm{H}, \alpha-\mathrm{CH}_{2}\right), 1.05\left(\mathrm{t}, 6 \mathrm{H}, \mathrm{CH}_{3}\right), 1.23\left(\mathrm{~m}, 4 \mathrm{H}, \gamma-\mathrm{CH}_{2}\right), 1.44\left(\mathrm{t}, 4 \mathrm{H} \beta-\mathrm{CH}_{2}\right), 1.84$ (s, $30 \mathrm{H}, \mathrm{Me}$-Cp) ppm., see Schock \& Marks (1988).

\section{S2. Refinement}

$\mathrm{H}$ atoms were placed in idealized positions with $\mathrm{d}(\mathrm{C}-\mathrm{H})=0.99 \AA\left(\mathrm{CH}_{2}\right), 0.98 \AA\left(\mathrm{CH}_{3}\right)$ and refined using a riding model with $U_{\text {iso }}(\mathrm{H})$ fixed at $1.2 U_{\mathrm{eq}}(\mathrm{C})$ for $\mathrm{CH}_{2}$ and $1.5 U_{\mathrm{eq}}(\mathrm{C})$ for $\mathrm{CH}_{3}$. The title compound crystallizes as racemic twin (Flack parameter $\mathrm{x}=0.471(5))$.
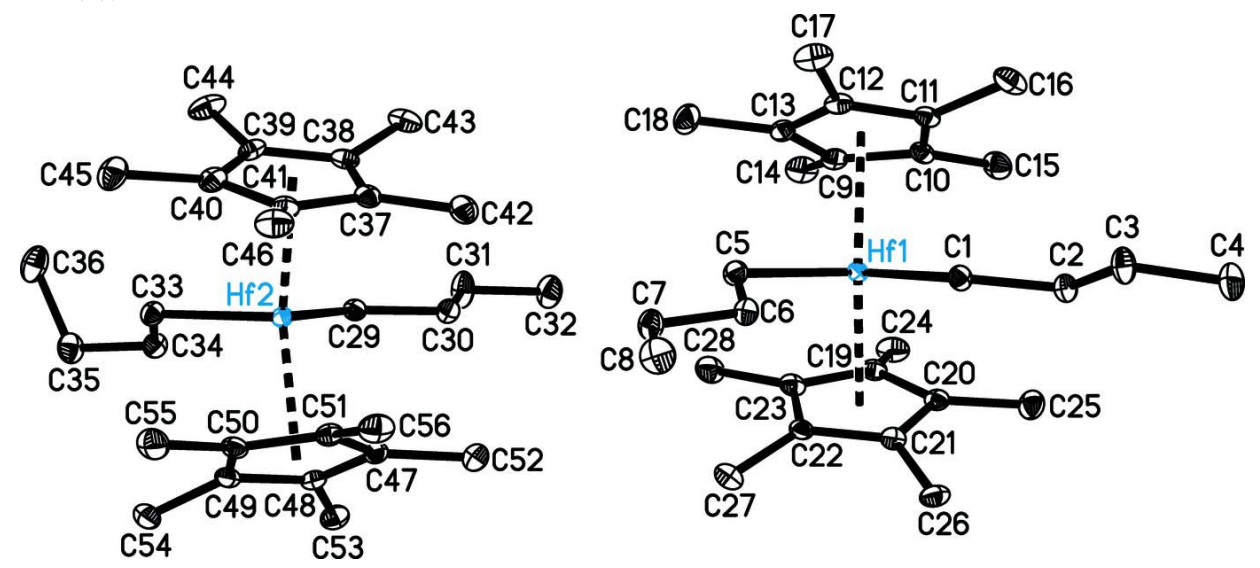

Figure 1

Molecular structure of the two molecules of the asymmetric unit with labelling and displacement ellipsoids drawn at $30 \%$ probability level. Hydrogen atoms are omitted for clarity.

Di- $n$-butylbis $\left(\eta^{5}\right.$-pentamethylcyclopentadienyl)hafnium(IV)

Crystal data

$\left[\mathrm{Hf}\left(\mathrm{C}_{10} \mathrm{H}_{15}\right)_{2}\left(\mathrm{C}_{4} \mathrm{H}_{9}\right)_{2}\right]$

$M_{r}=563.15$

Orthorhombic, $P 2_{1} 2_{1} 2_{1}$

$a=17.1572(3) \AA$

$$
\begin{aligned}
& b=17.2320(3) \AA \\
& c=17.2966(3) \AA \\
& V=5113.79(15) \AA^{3} \\
& Z=8
\end{aligned}
$$


$F(000)=2304$

$D_{\mathrm{x}}=1.463 \mathrm{Mg} \mathrm{m}^{-3}$

Mo $K \alpha$ radiation, $\lambda=0.71073 \AA$

Cell parameters from 9300 reflections

$\theta=2.4-28.7^{\circ}$

\section{Data collection}

\section{Bruker Kappa APEXII DUO CCD} diffractometer

Radiation source: fine-focus sealed tube Curved graphite monochromator Detector resolution: 8.3333 pixels $\mathrm{mm}^{-1}$ $\varphi$ and $\omega$ scans

Absorption correction: multi-scan (SADABS; Bruker, 2008)

$T_{\min }=0.30, T_{\max }=0.54$

\section{Refinement}

Refinement on $F^{2}$

Least-squares matrix: full

$R\left[F^{2}>2 \sigma\left(F^{2}\right)\right]=0.018$

$w R\left(F^{2}\right)=0.038$

$S=1.06$

13236 reflections

548 parameters

0 restraints

Primary atom site location: structure-invariant direct methods

Secondary atom site location: difference Fourier map $\mu=4.09 \mathrm{~mm}^{-1}$

$T=170 \mathrm{~K}$

Prism, colourless

$0.39 \times 0.27 \times 0.17 \mathrm{~mm}$

123798 measured reflections

13236 independent reflections

12433 reflections with $I>2 \sigma(I)$

$R_{\text {int }}=0.037$

$\theta_{\max }=28.7^{\circ}, \theta_{\min }=1.7^{\circ}$

$h=-18 \rightarrow 23$

$k=-23 \rightarrow 23$

$l=-23 \rightarrow 23$

\section{Special details}

Geometry. All e.s.d.'s (except the e.s.d. in the dihedral angle between two 1.s. planes) are estimated using the full covariance matrix. The cell e.s.d.'s are taken into account individually in the estimation of e.s.d.'s in distances, angles and torsion angles; correlations between e.s.d.'s in cell parameters are only used when they are defined by crystal symmetry. An approximate (isotropic) treatment of cell e.s.d.'s is used for estimating e.s.d.'s involving 1.s. planes.

Refinement. Refinement of $F^{2}$ against ALL reflections. The weighted $R$-factor $w R$ and goodness of fit $S$ are based on $F^{2}$, conventional $R$-factors $R$ are based on $F$, with $F$ set to zero for negative $F^{2}$. The threshold expression of $F^{2}>\sigma\left(F^{2}\right)$ is used only for calculating $R$-factors $(\mathrm{gt})$ etc. and is not relevant to the choice of reflections for refinement. $R$-factors based on $F^{2}$ are statistically about twice as large as those based on $F$, and $R$ - factors based on ALL data will be even larger.

Fractional atomic coordinates and isotropic or equivalent isotropic displacement parameters $\left(\AA^{2}\right)$

\begin{tabular}{lllll}
\hline & $x$ & $y$ & $z$ & $U_{\text {iso }} * / U_{\text {eq }}$ \\
\hline C1 & $0.10245(14)$ & $1.04413(14)$ & $0.84536(16)$ & $0.0209(5)$ \\
H1A & 0.0775 & 1.0294 & 0.7959 & $0.025^{*}$ \\
H1B & 0.0711 & 1.0192 & 0.8865 & $0.025^{*}$ \\
C2 & $0.08748(16)$ & $1.13134(15)$ & $0.85402(17)$ & $0.0234(6)$ \\
H2A & 0.0962 & 1.1463 & 0.9086 & $0.028^{*}$ \\
H2B & 0.1257 & 1.1599 & 0.8220 & $0.028^{*}$ \\
C3 & $0.00596(16)$ & $1.15583(16)$ & $0.83060(19)$ & $0.0300(7)$ \\
H3A & -0.0322 & 1.1245 & 0.8602 & $0.036^{*}$ \\
H3B & -0.0015 & 1.1438 & 0.7751 & $0.036^{*}$
\end{tabular}




\begin{tabular}{|c|c|c|c|c|}
\hline $\mathrm{C} 4$ & $-0.0116(2)$ & $1.24120(17)$ & $0.8436(2)$ & $0.0377(8)$ \\
\hline $\mathrm{H} 4 \mathrm{~A}$ & 0.0234 & 1.2728 & 0.8118 & $0.057^{*}$ \\
\hline H4B & -0.0039 & 1.2540 & 0.8983 & $0.057^{*}$ \\
\hline $\mathrm{H} 4 \mathrm{C}$ & -0.0658 & 1.2519 & 0.8291 & $0.057^{*}$ \\
\hline $\mathrm{C} 5$ & $0.16087(15)$ & $0.86169(15)$ & $0.84278(16)$ & $0.0227(5)$ \\
\hline H5A & 0.1638 & 0.8424 & 0.7889 & $0.027 *$ \\
\hline H5B & 0.1925 & 0.8262 & 0.8750 & $0.027^{*}$ \\
\hline C6 & $0.07551(15)$ & $0.85520(15)$ & $0.86963(16)$ & $0.0211(5)$ \\
\hline H6A & 0.0699 & 0.8814 & 0.9202 & $0.025^{*}$ \\
\hline H6B & 0.0416 & 0.8825 & 0.8321 & $0.025^{*}$ \\
\hline $\mathrm{C} 7$ & $0.04807(17)$ & $0.77125(16)$ & $0.87731(18)$ & $0.0260(6)$ \\
\hline H7A & 0.0595 & 0.7435 & 0.8285 & $0.031 *$ \\
\hline H7B & 0.0781 & 0.7458 & 0.9191 & $0.031 *$ \\
\hline $\mathrm{C} 8$ & $-0.03837(18)$ & $0.76363(18)$ & $0.8950(2)$ & $0.0358(7)$ \\
\hline H8A & -0.0687 & 0.7876 & 0.8533 & $0.054^{*}$ \\
\hline H8B & -0.0500 & 0.7898 & 0.9440 & $0.054^{*}$ \\
\hline $\mathrm{H} 8 \mathrm{C}$ & -0.0521 & 0.7086 & 0.8991 & $0.054^{*}$ \\
\hline C9 & 0.32937 (14) & $0.98398(18)$ & $0.74945(14)$ & $0.0213(5)$ \\
\hline $\mathrm{C} 10$ & $0.29051(18)$ & $1.05690(15)$ & $0.74342(14)$ & $0.0211(5)$ \\
\hline C11 & $0.21592(19)$ & 1.04259 (13) & 0.71054 (13) & $0.0211(5)$ \\
\hline $\mathrm{C} 12$ & $0.20807(17)$ & $0.96162(15)$ & $0.69862(14)$ & $0.0227(5)$ \\
\hline $\mathrm{C} 13$ & $0.27865(19)$ & $0.92560(15)$ & $0.72268(14)$ & $0.0232(5)$ \\
\hline $\mathrm{C} 14$ & $0.41568(15)$ & $0.9711(2)$ & $0.76112(17)$ & $0.0330(7)$ \\
\hline H14A & 0.4400 & 0.9582 & 0.7115 & $0.049 *$ \\
\hline H14B & 0.4236 & 0.9282 & 0.7976 & $0.049^{*}$ \\
\hline $\mathrm{H} 14 \mathrm{C}$ & 0.4394 & 1.0184 & 0.7819 & $0.049^{*}$ \\
\hline C15 & $0.32455(19)$ & $1.13609(18)$ & 0.75601 (19) & $0.0328(7)$ \\
\hline H15A & 0.2975 & 1.1616 & 0.7990 & $0.049 *$ \\
\hline H15B & 0.3183 & 1.1671 & 0.7089 & $0.049 *$ \\
\hline $\mathrm{H} 15 \mathrm{C}$ & 0.3801 & 1.1313 & 0.7683 & $0.049^{*}$ \\
\hline $\mathrm{C} 16$ & $0.16141(18)$ & $1.10256(19)$ & $0.67941(18)$ & $0.0342(7)$ \\
\hline H16A & 0.1647 & 1.1033 & 0.6228 & $0.051^{*}$ \\
\hline H16B & 0.1758 & 1.1536 & 0.6998 & $0.051^{*}$ \\
\hline $\mathrm{H} 16 \mathrm{C}$ & 0.1080 & 1.0900 & 0.6951 & $0.051 *$ \\
\hline $\mathrm{C} 17$ & $0.13977(18)$ & $0.9248(2)$ & 0.65925 (18) & $0.0360(7)$ \\
\hline H17A & 0.1460 & 0.8682 & 0.6596 & $0.054^{*}$ \\
\hline H17B & 0.1368 & 0.9432 & 0.6057 & $0.054 *$ \\
\hline $\mathrm{H} 17 \mathrm{C}$ & 0.0918 & 0.9387 & 0.6867 & $0.054^{*}$ \\
\hline $\mathrm{C} 18$ & 0.29965 (19) & $0.84198(17)$ & $0.71220(19)$ & $0.0365(8)$ \\
\hline $\mathrm{H} 18 \mathrm{~A}$ & 0.3252 & 0.8227 & 0.7591 & $0.055^{*}$ \\
\hline H18B & 0.3353 & 0.8367 & 0.6682 & $0.055^{*}$ \\
\hline $\mathrm{H} 18 \mathrm{C}$ & 0.2523 & 0.8117 & 0.7024 & $0.055^{*}$ \\
\hline C19 & $0.32414(14)$ & 1.02052 (19) & 0.94309 (14) & $0.0225(5)$ \\
\hline $\mathrm{C} 20$ & $0.25657(15)$ & $1.06311(16)$ & $0.96461(15)$ & $0.0212(5)$ \\
\hline $\mathrm{C} 21$ & 0.20008 (14) & $1.00970(16)$ & $0.99217(15)$ & $0.0220(6)$ \\
\hline $\mathrm{C} 22$ & $0.23236(15)$ & $0.93384(15)$ & 0.98649 (14) & $0.0203(6)$ \\
\hline $\mathrm{C} 23$ & $0.30815(15)$ & $0.94104(17)$ & $0.95437(16)$ & $0.0225(6)$ \\
\hline C24 & $0.40301(17)$ & $1.05688(19)$ & $0.93168(18)$ & $0.0334(7)$ \\
\hline
\end{tabular}




\begin{tabular}{|c|c|c|c|c|}
\hline $\mathrm{H} 24 \mathrm{~A}$ & 0.4402 & 1.0171 & 0.9148 & $0.050^{*}$ \\
\hline H24B & 0.4208 & 1.0796 & 0.9805 & $0.050^{*}$ \\
\hline $\mathrm{H} 24 \mathrm{C}$ & 0.3995 & 1.0976 & 0.8923 & $0.050^{*}$ \\
\hline $\mathrm{C} 25$ & $0.25347(18)$ & $1.14995(16)$ & $0.97023(19)$ & $0.0315(7)$ \\
\hline $\mathrm{H} 25 \mathrm{~A}$ & 0.2059 & 1.1655 & 0.9975 & $0.047^{*}$ \\
\hline H25B & 0.2534 & 1.1724 & 0.9182 & $0.047^{*}$ \\
\hline $\mathrm{H} 25 \mathrm{C}$ & 0.2991 & 1.1687 & 0.9987 & $0.047^{*}$ \\
\hline $\mathrm{C} 26$ & $0.12593(15)$ & 1.02885 (19) & $1.03341(15)$ & $0.0274(6)$ \\
\hline $\mathrm{H} 26 \mathrm{~A}$ & 0.1121 & 1.0831 & 1.0236 & $0.041^{*}$ \\
\hline $\mathrm{H} 26 \mathrm{~B}$ & 0.1330 & 1.0209 & 1.0891 & $0.041^{*}$ \\
\hline $\mathrm{H} 26 \mathrm{C}$ & 0.0841 & 0.9950 & 1.0146 & $0.041^{*}$ \\
\hline C27 & $0.19842(17)$ & $0.86248(17)$ & $1.02273(17)$ & 0.0300 \\
\hline $\mathrm{H} 27 \mathrm{~A}$ & 0.1468 & 0.8524 & 1.0006 & $0.045^{*}$ \\
\hline $\mathrm{H} 27 \mathrm{~B}$ & 0.1936 & 0.8703 & 1.0786 & $0.045^{*}$ \\
\hline $\mathrm{H} 27 \mathrm{C}$ & 0.2327 & 0.8181 & 1.0127 & $0.045^{*}$ \\
\hline C28 & $0.36408(18)$ & $0.87419(18)$ & $0.94451(18)$ & 0.0330 \\
\hline $\mathrm{H} 28 \mathrm{~A}$ & 0.4141 & 0.8937 & 0.9254 & $0.049^{*}$ \\
\hline $\mathrm{H} 28 \mathrm{~B}$ & 0.3425 & 0.8370 & 0.9073 & $0.049^{*}$ \\
\hline $\mathrm{H} 28 \mathrm{C}$ & 0.3719 & 0.8484 & 0.9944 & $0.049 *$ \\
\hline C29 & $0.39487(15)$ & $0.54682(14)$ & $0.88712(16)$ & $0.0206(5)$ \\
\hline $\mathrm{H} 29 \mathrm{~A}$ & 0.4240 & 0.5259 & 0.9319 & $0.025^{*}$ \\
\hline H29B & 0.4238 & 0.5293 & 0.8408 & $0.025^{*}$ \\
\hline C30 & $0.40920(15)$ & $0.63443(15)$ & $0.89038(17)$ & $0.0229(5)$ \\
\hline $\mathrm{H} 30 \mathrm{~A}$ & 0.3763 & 0.6603 & 0.8511 & $0.028 *$ \\
\hline H30B & 0.3934 & 0.6541 & 0.9418 & $0.028^{*}$ \\
\hline C31 & $0.49422(17)$ & 0.65535 (17) & $0.8760(2)$ & 0.0368 \\
\hline $\mathrm{H} 31 \mathrm{~A}$ & 0.5268 & 0.6278 & 0.9145 & $0.044 *$ \\
\hline H31B & 0.5092 & 0.6361 & 0.8242 & $0.044^{*}$ \\
\hline C32 & 0.5129 (2) & $0.74127(17)$ & $0.8802(2)$ & $0.0395(8)$ \\
\hline $\mathrm{H} 32 \mathrm{~A}$ & 0.4989 & 0.7612 & 0.9315 & $0.059 *$ \\
\hline H32B & 0.4832 & 0.7691 & 0.8406 & $0.059^{*}$ \\
\hline $\mathrm{H} 32 \mathrm{C}$ & 0.5688 & 0.7491 & 0.8714 & $0.059^{*}$ \\
\hline C33 & $0.35133(16)$ & $0.36285(15)$ & $0.88524(16)$ & 0.0230 \\
\hline $\mathrm{H} 33 \mathrm{~A}$ & 0.3197 & 0.3240 & 0.9134 & $0.028^{*}$ \\
\hline H33B & 0.3559 & 0.3447 & 0.8311 & $0.028^{*}$ \\
\hline C34 & $0.43333(16)$ & $0.36327(16)$ & $0.92074(17)$ & $0.0257(6)$ \\
\hline $\mathrm{H} 34 \mathrm{~A}$ & 0.4666 & 0.3985 & 0.8898 & $0.031 *$ \\
\hline H34B & 0.4299 & 0.3851 & 0.9736 & $0.031^{*}$ \\
\hline C35 & $0.47354(18)$ & $0.28392(17)$ & $0.9256(2)$ & $0.0334(7)$ \\
\hline $\mathrm{H} 35 \mathrm{~A}$ & 0.5219 & 0.2892 & 0.9564 & $0.040^{*}$ \\
\hline H35B & 0.4388 & 0.2472 & 0.9530 & $0.040^{*}$ \\
\hline C36 & 0.4937 (2) & 0.25057 (19) & $0.8475(2)$ & $0.0411(8)$ \\
\hline H36A & 0.5313 & 0.2846 & 0.8215 & $0.062 *$ \\
\hline H36B & 0.4464 & 0.2466 & 0.8160 & $0.062^{*}$ \\
\hline $\mathrm{H} 36 \mathrm{C}$ & 0.5166 & 0.1989 & 0.8541 & $0.062 *$ \\
\hline C37 & $0.2159(2)$ & $0.55026(15)$ & 0.77661 (14) & 0.0228 \\
\hline $\mathrm{C} 38$ & $0.29141(17)$ & $0.53422(15)$ & $0.74613(13)$ & $0.0220(6)$ \\
\hline C39 & $0.29843(15)$ & $0.45287(16)$ & $0.73771(15)$ & $0.0227(6)$ \\
\hline
\end{tabular}




\begin{tabular}{|c|c|c|c|c|}
\hline $\mathrm{C} 40$ & $0.22729(18)$ & $0.41820(16)$ & $0.76189(16)$ & $0.0241(6)$ \\
\hline $\mathrm{C} 41$ & $0.17574(14)$ & 0.47824 (19) & $0.78436(14)$ & $0.0225(5)$ \\
\hline $\mathrm{C} 42$ & $0.18260(18)$ & $0.63014(17)$ & $0.78753(18)$ & $0.0305(7)$ \\
\hline $\mathrm{H} 42 \mathrm{~A}$ & 0.1660 & 0.6509 & 0.7374 & $0.046^{*}$ \\
\hline $\mathrm{H} 42 \mathrm{~B}$ & 0.2224 & 0.6642 & 0.8099 & $0.046^{*}$ \\
\hline $\mathrm{H} 42 \mathrm{C}$ & 0.1377 & 0.6274 & 0.8224 & $0.046^{*}$ \\
\hline $\mathrm{C} 43$ & 0.34800 (19) & 0.59149 (19) & $0.71299(17)$ & $0.0331(7)$ \\
\hline $\mathrm{H} 43 \mathrm{~A}$ & 0.3474 & 0.5879 & 0.6565 & $0.050 *$ \\
\hline $\mathrm{H} 43 \mathrm{~B}$ & 0.4005 & 0.5800 & 0.7321 & $0.050 *$ \\
\hline $\mathrm{H} 43 \mathrm{C}$ & 0.3331 & 0.6441 & 0.7287 & $0.050^{*}$ \\
\hline $\mathrm{C} 44$ & $0.36646(18)$ & 0.4137 (2) & 0.70034 (19) & $0.0387(8)$ \\
\hline $\mathrm{H} 44 \mathrm{~A}$ & 0.4142 & 0.4271 & 0.7284 & $0.058^{*}$ \\
\hline H44B & 0.3709 & 0.4311 & 0.6465 & $0.058^{*}$ \\
\hline $\mathrm{H} 44 \mathrm{C}$ & 0.3589 & 0.3574 & 0.7016 & $0.058^{*}$ \\
\hline $\mathrm{C} 45$ & 0.2069 (2) & 0.33404 (17) & $0.75571(19)$ & $0.0361(7)$ \\
\hline $\mathrm{H} 45 \mathrm{~A}$ & 0.1969 & 0.3209 & 0.7015 & $0.054 *$ \\
\hline $\mathrm{H} 45 \mathrm{~B}$ & 0.1601 & 0.3234 & 0.7865 & $0.054 *$ \\
\hline $\mathrm{H} 45 \mathrm{C}$ & 0.2503 & 0.3026 & 0.7751 & $0.054 *$ \\
\hline $\mathrm{C} 46$ & $0.08939(16)$ & $0.4674(2)$ & 0.79405 (17) & $0.0331(7)$ \\
\hline $\mathrm{H} 46 \mathrm{~A}$ & 0.0796 & 0.4268 & 0.8325 & $0.050 *$ \\
\hline H46B & 0.0664 & 0.4521 & 0.7445 & $0.050^{*}$ \\
\hline $\mathrm{H} 46 \mathrm{C}$ & 0.0659 & 0.5162 & 0.8115 & $0.050 *$ \\
\hline $\mathrm{C} 47$ & $0.23728(15)$ & $0.55693(16)$ & $1.00118(15)$ & $0.0209(5)$ \\
\hline $\mathrm{C} 48$ & $0.29283(17)$ & $0.50411(14)$ & $1.03097(14)$ & $0.0218(6)$ \\
\hline $\mathrm{C} 49$ & $0.26283(16)$ & $0.42810(16)$ & $1.02337(15)$ & $0.0230(6)$ \\
\hline $\mathrm{C} 50$ & $0.18791(16)$ & $0.43383(17)$ & $0.98829(16)$ & $0.0221(6)$ \\
\hline $\mathrm{C} 51$ & $0.17114(15)$ & $0.51383(18)$ & $0.97676(15)$ & $0.0222(5)$ \\
\hline $\mathrm{C} 52$ & $0.23970(17)$ & $0.64415(16)$ & $1.00693(18)$ & $0.0308(7)$ \\
\hline $\mathrm{H} 52 \mathrm{~A}$ & 0.2876 & 0.6601 & 1.0334 & $0.046^{*}$ \\
\hline H52B & 0.1944 & 0.6625 & 1.0363 & $0.046^{*}$ \\
\hline $\mathrm{H} 52 \mathrm{C}$ & 0.2387 & 0.6666 & 0.9549 & $0.046^{*}$ \\
\hline C53 & $0.36538(15)$ & $0.52551(18)$ & $1.07501(16)$ & $0.0293(6)$ \\
\hline H53A & 0.4056 & 0.4862 & 1.0660 & $0.044 *$ \\
\hline H53B & 0.3535 & 0.5281 & 1.1304 & $0.044^{*}$ \\
\hline $\mathrm{H} 53 \mathrm{C}$ & 0.3843 & 0.5762 & 1.0573 & $0.044^{*}$ \\
\hline C54 & $0.29470(18)$ & $0.35685(16)$ & $1.06056(16)$ & 0.0299 (6) \\
\hline H54A & 0.2809 & 0.3566 & 1.1155 & $0.045^{*}$ \\
\hline H54B & 0.3516 & 0.3562 & 1.0552 & $0.045^{*}$ \\
\hline $\mathrm{H} 54 \mathrm{C}$ & 0.2727 & 0.3108 & 1.0354 & $0.045^{*}$ \\
\hline C55 & 0.13305 (19) & $0.36651(18)$ & $0.97753(19)$ & $0.0355(7)$ \\
\hline H55A & 0.0846 & 0.3849 & 0.9539 & $0.053^{*}$ \\
\hline H55B & 0.1215 & 0.3431 & 1.0279 & $0.053 *$ \\
\hline $\mathrm{H} 55 \mathrm{C}$ & 0.1574 & 0.3277 & 0.9439 & $0.053 *$ \\
\hline C56 & $0.09175(17)$ & $0.54926(19)$ & $0.96328(18)$ & $0.0330(7)$ \\
\hline H56A & 0.0955 & 0.5892 & 0.9231 & $0.049^{*}$ \\
\hline H56B & 0.0729 & 0.5727 & 1.0114 & $0.049^{*}$ \\
\hline $\mathrm{H} 56 \mathrm{C}$ & 0.0553 & 0.5088 & 0.9466 & $0.049^{*}$ \\
\hline Hf1 & $0.218019(6)$ & $0.981329(5)$ & $0.846352(5)$ & $0.01504(2)$ \\
\hline
\end{tabular}




$\begin{array}{lllll}\mathrm{H} 2 & 0.283647(6) & 0.477447(5) & 0.884701(5) & 0.01551(2)\end{array}$

Atomic displacement parameters $\left(\AA^{2}\right)$

\begin{tabular}{|c|c|c|c|c|c|c|}
\hline & $U^{11}$ & $U^{22}$ & $U^{33}$ & $U^{12}$ & $U^{13}$ & $U^{23}$ \\
\hline $\mathrm{C} 1$ & $0.0178(12)$ & 0.0213 (12) & $0.0235(13)$ & $0.0033(9)$ & $0.0016(10)$ & $0.0020(10)$ \\
\hline $\mathrm{C} 2$ & $0.0213(14)$ & $0.0206(13)$ & $0.0282(15)$ & $0.0014(10)$ & $-0.0010(11)$ & $0.0015(11)$ \\
\hline $\mathrm{C} 3$ & $0.0211(14)$ & $0.0229(14)$ & 0.0461 (19) & $0.0044(11)$ & $-0.0013(13)$ & $0.0031(12)$ \\
\hline $\mathrm{C} 4$ & 0.0388 (19) & $0.0273(15)$ & $0.047(2)$ & $0.0118(13)$ & $-0.0021(16)$ & $0.0025(14)$ \\
\hline $\mathrm{C} 5$ & 0.0247 (14) & $0.0205(12)$ & $0.0228(13)$ & $-0.0034(10)$ & $0.0026(11)$ & $-0.0011(10)$ \\
\hline C6 & $0.0182(13)$ & $0.0200(12)$ & $0.0253(14)$ & $-0.0014(10)$ & $-0.0015(10)$ & $-0.0014(10)$ \\
\hline $\mathrm{C} 7$ & $0.0260(15)$ & $0.0201(12)$ & $0.0320(15)$ & $-0.0040(11)$ & 0.0008 (12) & $-0.0015(12)$ \\
\hline $\mathrm{C} 8$ & $0.0285(16)$ & $0.0370(16)$ & 0.0421 (19) & $-0.0115(13)$ & $0.0016(13)$ & $-0.0002(14)$ \\
\hline $\mathrm{C} 9$ & $0.0154(12)$ & $0.0299(13)$ & $0.0184(11)$ & 0.0004 (12) & $0.0061(9)$ & $0.0006(12)$ \\
\hline $\mathrm{C} 10$ & $0.0201(14)$ & $0.0246(12)$ & $0.0187(12)$ & 0.0008 (12) & $0.0033(11)$ & $0.0057(10)$ \\
\hline C11 & $0.0186(11)$ & $0.0284(13)$ & $0.0163(10)$ & $0.0020(13)$ & $0.0021(11)$ & $0.0043(9)$ \\
\hline $\mathrm{C} 12$ & $0.0212(14)$ & 0.0308 (14) & $0.0162(11)$ & $-0.0028(11)$ & $0.0019(10)$ & $-0.0011(9)$ \\
\hline $\mathrm{C} 13$ & 0.0249 (13) & $0.0266(12)$ & $0.0182(11)$ & $0.0042(13)$ & $0.0074(12)$ & $-0.0006(9)$ \\
\hline C14 & $0.0167(13)$ & 0.0515 (19) & $0.0309(15)$ & $0.0051(14)$ & $0.0056(11)$ & $0.0018(15)$ \\
\hline $\mathrm{C} 15$ & $0.0354(17)$ & $0.0306(16)$ & $0.0323(17)$ & $-0.0080(13)$ & 0.0027 (13) & 0.0057 (13) \\
\hline $\mathrm{C} 16$ & $0.0277(16)$ & $0.0476(18)$ & $0.0272(16)$ & $0.0102(14)$ & $0.0030(12)$ & $0.0139(14)$ \\
\hline $\mathrm{C} 17$ & $0.0293(16)$ & $0.052(2)$ & $0.0266(16)$ & $-0.0082(14)$ & $0.0015(13)$ & $-0.0051(14)$ \\
\hline $\mathrm{C} 18$ & $0.042(2)$ & $0.0298(15)$ & $0.0374(17)$ & 0.0064 (13) & $0.0169(14)$ & $-0.0036(12)$ \\
\hline C19 & $0.0205(12)$ & 0.0292 (13) & 0.0177 (12) & $-0.0007(12)$ & $-0.0047(9)$ & $0.0012(12)$ \\
\hline $\mathrm{C} 20$ & $0.0237(13)$ & $0.0228(13)$ & $0.0172(12)$ & $-0.0055(10)$ & $-0.0026(10)$ & $-0.0014(11)$ \\
\hline $\mathrm{C} 21$ & $0.0211(15)$ & $0.0256(13)$ & $0.0192(11)$ & $0.0000(10)$ & $0.0003(9)$ & $0.0012(10)$ \\
\hline $\mathrm{C} 22$ & $0.0223(16)$ & $0.0237(12)$ & $0.0149(11)$ & $0.0030(10)$ & $-0.0017(10)$ & $0.0026(10)$ \\
\hline $\mathrm{C} 23$ & $0.0176(13)$ & 0.0303 (14) & $0.0196(13)$ & $0.0046(11)$ & $-0.0046(10)$ & $0.0034(11)$ \\
\hline $\mathrm{C} 24$ & $0.0257(15)$ & $0.0470(17)$ & $0.0275(15)$ & $-0.0080(13)$ & $0.0011(12)$ & $0.0012(14)$ \\
\hline $\mathrm{C} 25$ & $0.0351(16)$ & 0.0253 (14) & $0.0341(16)$ & $-0.0023(12)$ & $-0.0048(13)$ & $-0.0049(13)$ \\
\hline $\mathrm{C} 26$ & $0.0275(14)$ & 0.0367 (16) & $0.0180(12)$ & $0.0036(13)$ & $0.0044(10)$ & $-0.0022(12)$ \\
\hline $\mathrm{C} 27$ & $0.0330(18)$ & 0.0307 (14) & $0.0262(14)$ & $-0.0011(12)$ & 0.0005 (12) & $0.0083(12)$ \\
\hline $\mathrm{C} 28$ & $0.0283(16)$ & $0.0400(17)$ & $0.0306(17)$ & $0.0106(13)$ & $-0.0013(12)$ & $0.0056(13)$ \\
\hline $\mathrm{C} 29$ & $0.0203(13)$ & $0.0224(12)$ & $0.0190(12)$ & $-0.0028(9)$ & $-0.0017(10)$ & $0.0002(10)$ \\
\hline $\mathrm{C} 30$ & $0.0205(13)$ & $0.0228(13)$ & $0.0255(14)$ & $-0.0014(10)$ & $0.0029(11)$ & $-0.0019(11)$ \\
\hline $\mathrm{C} 31$ & $0.0221(15)$ & $0.0233(14)$ & $0.065(2)$ & $-0.0049(11)$ & $0.0082(15)$ & $-0.0028(14)$ \\
\hline $\mathrm{C} 32$ & 0.0367 (19) & $0.0283(16)$ & $0.054(2)$ & $-0.0094(14)$ & $0.0142(16)$ & $-0.0030(16)$ \\
\hline C33 & $0.0229(13)$ & $0.0222(12)$ & $0.0237(13)$ & $0.0025(10)$ & $-0.0013(11)$ & $-0.0024(11)$ \\
\hline C34 & $0.0251(14)$ & $0.0254(13)$ & 0.0267 (14) & $0.0019(11)$ & $-0.0019(11)$ & $-0.0012(12)$ \\
\hline $\mathrm{C} 35$ & 0.0308 (16) & $0.0292(15)$ & $0.0404(17)$ & 0.0067 (12) & $-0.0054(14)$ & $0.0024(13)$ \\
\hline $\mathrm{C} 36$ & $0.0299(18)$ & 0.0337 (17) & $0.060(2)$ & $0.0034(13)$ & $0.0035(17)$ & $-0.0128(15)$ \\
\hline $\mathrm{C} 37$ & $0.0228(13)$ & $0.0270(12)$ & $0.0186(11)$ & $0.0028(13)$ & $-0.0065(13)$ & $0.0026(9)$ \\
\hline C38 & 0.0195 (13) & $0.0318(14)$ & $0.0147(10)$ & $-0.0019(12)$ & $-0.0026(10)$ & $0.0036(10)$ \\
\hline C39 & $0.0214(16)$ & 0.0298 (14) & $0.0168(11)$ & $0.0043(10)$ & $-0.0029(10)$ & $-0.0069(10)$ \\
\hline $\mathrm{C} 40$ & $0.0204(15)$ & $0.0301(14)$ & $0.0219(12)$ & $-0.0012(12)$ & $-0.0085(12)$ & $-0.0040(10)$ \\
\hline $\mathrm{C} 41$ & 0.0195 (12) & $0.0298(13)$ & $0.0182(12)$ & $0.0013(12)$ & $-0.0046(9)$ & 0.0013 (12) \\
\hline $\mathrm{C} 42$ & $0.0312(16)$ & $0.0313(16)$ & $0.0289(16)$ & $0.0069(12)$ & $-0.0045(13)$ & $0.0040(12)$ \\
\hline $\mathrm{C} 43$ & $0.0319(16)$ & $0.0455(18)$ & $0.0217(14)$ & $-0.0090(14)$ & $-0.0019(12)$ & $0.0091(13)$ \\
\hline
\end{tabular}




\begin{tabular}{lllllll} 
C44 & $0.0329(18)$ & $0.058(2)$ & $0.0251(16)$ & $0.0102(15)$ & $0.0009(13)$ & $-0.0147(15)$ \\
C45 & $0.0346(19)$ & $0.0340(15)$ & $0.0397(17)$ & $-0.0056(14)$ & $-0.0096(15)$ & $-0.0088(13)$ \\
C46 & $0.0222(14)$ & $0.051(2)$ & $0.0265(14)$ & $-0.0042(13)$ & $-0.0032(11)$ & $-0.0011(14)$ \\
C47 & $0.0236(15)$ & $0.0219(12)$ & $0.0171(12)$ & $-0.0018(10)$ & $0.0040(10)$ & $-0.0026(10)$ \\
C48 & $0.0253(15)$ & $0.0260(13)$ & $0.0142(10)$ & $-0.0001(10)$ & $0.0009(10)$ & $-0.0033(9)$ \\
C49 & $0.0287(17)$ & $0.0255(13)$ & $0.0149(12)$ & $-0.0028(11)$ & $0.0011(10)$ & $0.0020(10)$ \\
C50 & $0.0233(14)$ & $0.0259(14)$ & $0.0172(13)$ & $-0.0041(11)$ & $0.0037(10)$ & $0.0028(11)$ \\
C51 & $0.0197(12)$ & $0.0283(14)$ & $0.0187(12)$ & $0.0012(12)$ & $0.0037(9)$ & $-0.0017(12)$ \\
C52 & $0.0359(18)$ & $0.0273(14)$ & $0.0294(15)$ & $0.0046(12)$ & $0.0036(12)$ & $-0.0029(12)$ \\
C53 & $0.0293(14)$ & $0.0351(15)$ & $0.0235(13)$ & $0.0000(13)$ & $-0.0042(11)$ & $-0.0049(14)$ \\
C54 & $0.0364(18)$ & $0.0285(13)$ & $0.0249(14)$ & $0.0020(12)$ & $0.0018(12)$ & $0.0061(11)$ \\
C55 & $0.0353(18)$ & $0.0373(17)$ & $0.0337(18)$ & $-0.0143(14)$ & $0.0021(14)$ & $0.0019(14)$ \\
C56 & $0.0235(15)$ & $0.0464(18)$ & $0.0289(15)$ & $0.0084(13)$ & $0.0042(12)$ & $-0.0011(13)$ \\
Hf1 & $0.01256(4)$ & $0.01658(4)$ & $0.01597(4)$ & $0.00068(5)$ & $0.00096(4)$ & $0.00147(4)$ \\
Hf2 & $0.01422(4)$ & $0.01740(4)$ & $0.01492(4)$ & $-0.00044(6)$ & $-0.00092(4)$ & $-0.00109(4)$ \\
& & & & & & \\
\hline
\end{tabular}

Geometric parameters $\left(\hat{A},{ }^{o}\right)$

\begin{tabular}{|c|c|c|c|}
\hline $\mathrm{C} 1-\mathrm{C} 2$ & $1.532(3)$ & $\mathrm{C} 29-\mathrm{C} 30$ & $1.531(3)$ \\
\hline $\mathrm{C} 1-\mathrm{Hf1}$ & $2.259(2)$ & $\mathrm{C} 29-\mathrm{Hf} 2$ & $2.252(2)$ \\
\hline $\mathrm{C} 1-\mathrm{H} 1 \mathrm{~A}$ & 0.9900 & $\mathrm{C} 29-\mathrm{H} 29 \mathrm{~A}$ & 0.9900 \\
\hline $\mathrm{C} 1-\mathrm{H} 1 \mathrm{~B}$ & 0.9900 & $\mathrm{C} 29-\mathrm{H} 29 \mathrm{~B}$ & 0.9900 \\
\hline $\mathrm{C} 2-\mathrm{C} 3$ & $1.516(4)$ & $\mathrm{C} 30-\mathrm{C} 31$ & $1.523(4)$ \\
\hline $\mathrm{C} 2-\mathrm{H} 2 \mathrm{~A}$ & 0.9900 & $\mathrm{C} 30-\mathrm{H} 30 \mathrm{~A}$ & 0.9900 \\
\hline $\mathrm{C} 2-\mathrm{H} 2 \mathrm{~B}$ & 0.9900 & $\mathrm{C} 30-\mathrm{H} 30 \mathrm{~B}$ & 0.9900 \\
\hline $\mathrm{C} 3-\mathrm{C} 4$ & $1.518(4)$ & $\mathrm{C} 31-\mathrm{C} 32$ & $1.517(4)$ \\
\hline $\mathrm{C} 3-\mathrm{H} 3 \mathrm{~A}$ & 0.9900 & $\mathrm{C} 31-\mathrm{H} 31 \mathrm{~A}$ & 0.9900 \\
\hline C $3-\mathrm{H} 3 \mathrm{~B}$ & 0.9900 & $\mathrm{C} 31-\mathrm{H} 31 \mathrm{~B}$ & 0.9900 \\
\hline $\mathrm{C} 4-\mathrm{H} 4 \mathrm{~A}$ & 0.9800 & $\mathrm{C} 32-\mathrm{H} 32 \mathrm{~A}$ & 0.9800 \\
\hline $\mathrm{C} 4-\mathrm{H} 4 \mathrm{~B}$ & 0.9800 & $\mathrm{C} 32-\mathrm{H} 32 \mathrm{~B}$ & 0.9800 \\
\hline $\mathrm{C} 4-\mathrm{H} 4 \mathrm{C}$ & 0.9800 & $\mathrm{C} 32-\mathrm{H} 32 \mathrm{C}$ & 0.9800 \\
\hline $\mathrm{C} 5-\mathrm{C} 6$ & $1.540(4)$ & $\mathrm{C} 33-\mathrm{C} 34$ & $1.535(4)$ \\
\hline C5-Hf1 & $2.284(2)$ & C33-Hf2 & $2.291(3)$ \\
\hline $\mathrm{C} 5-\mathrm{H} 5 \mathrm{~A}$ & 0.9900 & $\mathrm{C} 33-\mathrm{H} 33 \mathrm{~A}$ & 0.9900 \\
\hline C5-H5B & 0.9900 & C $33-\mathrm{H} 33 \mathrm{~B}$ & 0.9900 \\
\hline $\mathrm{C} 6-\mathrm{C} 7$ & $1.527(4)$ & $\mathrm{C} 34-\mathrm{C} 35$ & $1.534(4)$ \\
\hline C6-H6A & 0.9900 & $\mathrm{C} 34-\mathrm{H} 34 \mathrm{~A}$ & 0.9900 \\
\hline C6-H6B & 0.9900 & C34-H34B & 0.9900 \\
\hline $\mathrm{C} 7-\mathrm{C} 8$ & $1.520(4)$ & $\mathrm{C} 35-\mathrm{C} 36$ & $1.509(5)$ \\
\hline C7-H7A & 0.9900 & C35-H35A & 0.9900 \\
\hline C7-H7B & 0.9900 & C35-H35B & 0.9900 \\
\hline $\mathrm{C} 8-\mathrm{H} 8 \mathrm{~A}$ & 0.9800 & C $36-\mathrm{H} 36 \mathrm{~A}$ & 0.9800 \\
\hline $\mathrm{C} 8-\mathrm{H} 8 \mathrm{~B}$ & 0.9800 & $\mathrm{C} 36-\mathrm{H} 36 \mathrm{~B}$ & 0.9800 \\
\hline $\mathrm{C} 8-\mathrm{H} 8 \mathrm{C}$ & 0.9800 & $\mathrm{C} 36-\mathrm{H} 36 \mathrm{C}$ & 0.9800 \\
\hline $\mathrm{C} 9-\mathrm{C} 13$ & $1.408(4)$ & C37-C38 & $1.425(4)$ \\
\hline $\mathrm{C} 9-\mathrm{C} 10$ & $1.426(4)$ & $\mathrm{C} 37-\mathrm{C} 41$ & $1.426(4)$ \\
\hline $\mathrm{C} 9-\mathrm{C} 14$ & $1.511(3)$ & $\mathrm{C} 37-\mathrm{C} 42$ & $1.502(4)$ \\
\hline C9-Hf1 & $2.542(2)$ & C37-Hf2 & $2.534(3)$ \\
\hline
\end{tabular}




\begin{tabular}{|c|c|c|c|}
\hline $\mathrm{C} 10-\mathrm{C} 11$ & $1.422(4)$ & C $38-\mathrm{C} 39$ & $1.415(4)$ \\
\hline $\mathrm{C} 10-\mathrm{C} 15$ & $1.500(4)$ & $\mathrm{C} 38-\mathrm{C} 43$ & $1.498(4)$ \\
\hline C10-Hf1 & $2.532(3)$ & C38-Hf2 & $2.592(2)$ \\
\hline $\mathrm{C} 11-\mathrm{C} 12$ & $1.417(4)$ & $\mathrm{C} 39-\mathrm{C} 40$ & $1.422(4)$ \\
\hline $\mathrm{C} 11-\mathrm{C} 16$ & $1.494(4)$ & $\mathrm{C} 39-\mathrm{C} 44$ & $1.495(4)$ \\
\hline C11-Hf1 & $2.576(2)$ & C39-Hf2 & $2.590(3)$ \\
\hline $\mathrm{C} 12-\mathrm{C} 13$ & $1.423(4)$ & $\mathrm{C} 40-\mathrm{C} 41$ & $1.416(4)$ \\
\hline $\mathrm{C} 12-\mathrm{C} 17$ & $1.497(4)$ & $\mathrm{C} 40-\mathrm{C} 45$ & $1.496(4)$ \\
\hline $\mathrm{C} 12-\mathrm{Hf} 1$ & $2.583(2)$ & $\mathrm{C} 40-\mathrm{Hf} 2$ & $2.547(3)$ \\
\hline $\mathrm{C} 13-\mathrm{C} 18$ & $1.496(4)$ & $\mathrm{C} 41-\mathrm{C} 46$ & $1.503(4)$ \\
\hline C13-Hf1 & $2.565(2)$ & $\mathrm{C} 41-\mathrm{Hf} 2$ & $2.538(2)$ \\
\hline $\mathrm{C} 14-\mathrm{H} 14 \mathrm{~A}$ & 0.9800 & $\mathrm{C} 42-\mathrm{H} 42 \mathrm{~A}$ & 0.9800 \\
\hline C14-H14B & 0.9800 & $\mathrm{C} 42-\mathrm{H} 42 \mathrm{~B}$ & 0.9800 \\
\hline $\mathrm{C} 14-\mathrm{H} 14 \mathrm{C}$ & 0.9800 & $\mathrm{C} 42-\mathrm{H} 42 \mathrm{C}$ & 0.9800 \\
\hline C15-H15A & 0.9800 & $\mathrm{C} 43-\mathrm{H} 43 \mathrm{~A}$ & 0.9800 \\
\hline C15-H15B & 0.9800 & $\mathrm{C} 43-\mathrm{H} 43 \mathrm{~B}$ & 0.9800 \\
\hline $\mathrm{C} 15-\mathrm{H} 15 \mathrm{C}$ & 0.9800 & $\mathrm{C} 43-\mathrm{H} 43 \mathrm{C}$ & 0.9800 \\
\hline $\mathrm{C} 16-\mathrm{H} 16 \mathrm{~A}$ & 0.9800 & $\mathrm{C} 44-\mathrm{H} 44 \mathrm{~A}$ & 0.9800 \\
\hline C16-H16B & 0.9800 & $\mathrm{C} 44-\mathrm{H} 44 \mathrm{~B}$ & 0.9800 \\
\hline $\mathrm{C} 16-\mathrm{H} 16 \mathrm{C}$ & 0.9800 & $\mathrm{C} 44-\mathrm{H} 44 \mathrm{C}$ & 0.9800 \\
\hline C17-H17A & 0.9800 & $\mathrm{C} 45-\mathrm{H} 45 \mathrm{~A}$ & 0.9800 \\
\hline C17-H17B & 0.9800 & $\mathrm{C} 45-\mathrm{H} 45 \mathrm{~B}$ & 0.9800 \\
\hline C17-H17C & 0.9800 & $\mathrm{C} 45-\mathrm{H} 45 \mathrm{C}$ & 0.9800 \\
\hline $\mathrm{C} 18-\mathrm{H} 18 \mathrm{~A}$ & 0.9800 & $\mathrm{C} 46-\mathrm{H} 46 \mathrm{~A}$ & 0.9800 \\
\hline C18-H18B & 0.9800 & $\mathrm{C} 46-\mathrm{H} 46 \mathrm{~B}$ & 0.9800 \\
\hline $\mathrm{C} 18-\mathrm{H} 18 \mathrm{C}$ & 0.9800 & $\mathrm{C} 46-\mathrm{H} 46 \mathrm{C}$ & 0.9800 \\
\hline $\mathrm{C} 19-\mathrm{C} 23$ & $1.410(4)$ & C47-C48 & $1.415(4)$ \\
\hline $\mathrm{C} 19-\mathrm{C} 20$ & $1.422(4)$ & $\mathrm{C} 47-\mathrm{C} 51$ & $1.421(4)$ \\
\hline $\mathrm{C} 19-\mathrm{C} 24$ & $1.504(4)$ & $\mathrm{C} 47-\mathrm{C} 52$ & $1.507(4)$ \\
\hline C19-Hf1 & $2.563(2)$ & C47-Hf2 & $2.563(3)$ \\
\hline $\mathrm{C} 20-\mathrm{C} 21$ & $1.419(4)$ & $\mathrm{C} 48-\mathrm{C} 49$ & $1.414(4)$ \\
\hline $\mathrm{C} 20-\mathrm{C} 25$ & $1.501(4)$ & $\mathrm{C} 48-\mathrm{C} 53$ & $1.505(4)$ \\
\hline $\mathrm{C} 20-\mathrm{Hf} 1$ & $2.571(3)$ & $\mathrm{C} 48-\mathrm{Hf} 2$ & $2.576(2)$ \\
\hline $\mathrm{C} 21-\mathrm{C} 22$ & $1.423(4)$ & $\mathrm{C} 49-\mathrm{C} 50$ & $1.425(4)$ \\
\hline $\mathrm{C} 21-\mathrm{C} 26$ & $1.496(3)$ & $\mathrm{C} 49-\mathrm{C} 54$ & $1.490(4)$ \\
\hline $\mathrm{C} 21-\mathrm{Hf} 1$ & $2.587(3)$ & C49-Hf2 & $2.570(3)$ \\
\hline $\mathrm{C} 22-\mathrm{C} 23$ & $1.419(4)$ & $\mathrm{C} 50-\mathrm{C} 51$ & $1.422(4)$ \\
\hline $\mathrm{C} 22-\mathrm{C} 27$ & $1.498(4)$ & $\mathrm{C} 50-\mathrm{C} 55$ & $1.505(4)$ \\
\hline $\mathrm{C} 22-\mathrm{Hf} 1$ & $2.570(2)$ & C50-Hf2 & $2.544(3)$ \\
\hline $\mathrm{C} 23-\mathrm{C} 28$ & 1.509 (4) & $\mathrm{C} 51-\mathrm{C} 56$ & $1.511(4)$ \\
\hline $\mathrm{C} 23-\mathrm{Hf} 1$ & $2.523(3)$ & C51—Hf2 & $2.580(2)$ \\
\hline $\mathrm{C} 24-\mathrm{H} 24 \mathrm{~A}$ & 0.9800 & $\mathrm{C} 52-\mathrm{H} 52 \mathrm{~A}$ & 0.9800 \\
\hline $\mathrm{C} 24-\mathrm{H} 24 \mathrm{~B}$ & 0.9800 & C52-H52B & 0.9800 \\
\hline $\mathrm{C} 24-\mathrm{H} 24 \mathrm{C}$ & 0.9800 & $\mathrm{C} 52-\mathrm{H} 52 \mathrm{C}$ & 0.9800 \\
\hline $\mathrm{C} 25-\mathrm{H} 25 \mathrm{~A}$ & 0.9800 & $\mathrm{C} 53-\mathrm{H} 53 \mathrm{~A}$ & 0.9800 \\
\hline $\mathrm{C} 25-\mathrm{H} 25 \mathrm{~B}$ & 0.9800 & C53-H53B & 0.9800 \\
\hline $\mathrm{C} 25-\mathrm{H} 25 \mathrm{C}$ & 0.9800 & $\mathrm{C} 53-\mathrm{H} 53 \mathrm{C}$ & 0.9800 \\
\hline $\mathrm{C} 26-\mathrm{H} 26 \mathrm{~A}$ & 0.9800 & $\mathrm{C} 54-\mathrm{H} 54 \mathrm{~A}$ & 0.9800 \\
\hline
\end{tabular}




\begin{tabular}{|c|c|c|c|}
\hline $\mathrm{C} 26-\mathrm{H} 26 \mathrm{~B}$ & 0.9800 & C54-H54B & 0.9800 \\
\hline $\mathrm{C} 26-\mathrm{H} 26 \mathrm{C}$ & 0.9800 & $\mathrm{C} 54-\mathrm{H} 54 \mathrm{C}$ & 0.9800 \\
\hline C27-H27A & 0.9800 & $\mathrm{C} 55-\mathrm{H} 55 \mathrm{~A}$ & 0.9800 \\
\hline $\mathrm{C} 27-\mathrm{H} 27 \mathrm{~B}$ & 0.9800 & C55-H55B & 0.9800 \\
\hline $\mathrm{C} 27-\mathrm{H} 27 \mathrm{C}$ & 0.9800 & $\mathrm{C} 55-\mathrm{H} 55 \mathrm{C}$ & 0.9800 \\
\hline $\mathrm{C} 28-\mathrm{H} 28 \mathrm{~A}$ & 0.9800 & $\mathrm{C} 56-\mathrm{H} 56 \mathrm{~A}$ & 0.9800 \\
\hline $\mathrm{C} 28-\mathrm{H} 28 \mathrm{~B}$ & 0.9800 & C56-H56B & 0.9800 \\
\hline $\mathrm{C} 28-\mathrm{H} 28 \mathrm{C}$ & 0.9800 & $\mathrm{C} 56-\mathrm{H} 56 \mathrm{C}$ & 0.9800 \\
\hline $\mathrm{C} 2-\mathrm{C} 1-\mathrm{Hf} 1$ & $128.05(18)$ & $\mathrm{C} 41-\mathrm{C} 40-\mathrm{C} 39$ & $108.1(2)$ \\
\hline $\mathrm{C} 2-\mathrm{C} 1-\mathrm{H} 1 \mathrm{~A}$ & 105.3 & $\mathrm{C} 41-\mathrm{C} 40-\mathrm{C} 45$ & $125.6(3)$ \\
\hline $\mathrm{Hf} 1-\mathrm{C} 1-\mathrm{H} 1 \mathrm{~A}$ & 105.3 & $\mathrm{C} 39-\mathrm{C} 40-\mathrm{C} 45$ & $126.0(3)$ \\
\hline $\mathrm{C} 2-\mathrm{C} 1-\mathrm{H} 1 \mathrm{~B}$ & 105.3 & $\mathrm{C} 41-\mathrm{C} 40-\mathrm{Hf} 2$ & $73.46(14)$ \\
\hline $\mathrm{Hf} 1-\mathrm{C} 1-\mathrm{H} 1 \mathrm{~B}$ & 105.3 & $\mathrm{C} 39-\mathrm{C} 40-\mathrm{Hf} 2$ & $75.58(15)$ \\
\hline $\mathrm{H} 1 \mathrm{~A}-\mathrm{C} 1-\mathrm{H} 1 \mathrm{~B}$ & 106.0 & $\mathrm{C} 45-\mathrm{C} 40-\mathrm{Hf} 2$ & $122.48(19)$ \\
\hline $\mathrm{C} 3-\mathrm{C} 2-\mathrm{C} 1$ & $113.7(2)$ & $\mathrm{C} 40-\mathrm{C} 41-\mathrm{C} 37$ & $107.9(2)$ \\
\hline $\mathrm{C} 3-\mathrm{C} 2-\mathrm{H} 2 \mathrm{~A}$ & 108.8 & $\mathrm{C} 40-\mathrm{C} 41-\mathrm{C} 46$ & $123.7(3)$ \\
\hline $\mathrm{C} 1-\mathrm{C} 2-\mathrm{H} 2 \mathrm{~A}$ & 108.8 & $\mathrm{C} 37-\mathrm{C} 41-\mathrm{C} 46$ & $126.6(3)$ \\
\hline $\mathrm{C} 3-\mathrm{C} 2-\mathrm{H} 2 \mathrm{~B}$ & 108.8 & $\mathrm{C} 40-\mathrm{C} 41-\mathrm{Hf} 2$ & $74.22(15)$ \\
\hline $\mathrm{C} 1-\mathrm{C} 2-\mathrm{H} 2 \mathrm{~B}$ & 108.8 & C $37-\mathrm{C} 41-\mathrm{Hf} 2$ & $73.51(14)$ \\
\hline $\mathrm{H} 2 \mathrm{~A}-\mathrm{C} 2-\mathrm{H} 2 \mathrm{~B}$ & 107.7 & $\mathrm{C} 46-\mathrm{C} 41-\mathrm{Hf} 2$ & $129.97(18)$ \\
\hline $\mathrm{C} 2-\mathrm{C} 3-\mathrm{C} 4$ & $114.4(3)$ & $\mathrm{C} 37-\mathrm{C} 42-\mathrm{H} 42 \mathrm{~A}$ & 109.5 \\
\hline $\mathrm{C} 2-\mathrm{C} 3-\mathrm{H} 3 \mathrm{~A}$ & 108.7 & $\mathrm{C} 37-\mathrm{C} 42-\mathrm{H} 42 \mathrm{~B}$ & 109.5 \\
\hline $\mathrm{C} 4-\mathrm{C} 3-\mathrm{H} 3 \mathrm{~A}$ & 108.7 & $\mathrm{H} 42 \mathrm{~A}-\mathrm{C} 42-\mathrm{H} 42 \mathrm{~B}$ & 109.5 \\
\hline $\mathrm{C} 2-\mathrm{C} 3-\mathrm{H} 3 \mathrm{~B}$ & 108.7 & $\mathrm{C} 37-\mathrm{C} 42-\mathrm{H} 42 \mathrm{C}$ & 109.5 \\
\hline $\mathrm{C} 4-\mathrm{C} 3-\mathrm{H} 3 \mathrm{~B}$ & 108.7 & $\mathrm{H} 42 \mathrm{~A}-\mathrm{C} 42-\mathrm{H} 42 \mathrm{C}$ & 109.5 \\
\hline $\mathrm{H} 3 \mathrm{~A}-\mathrm{C} 3-\mathrm{H} 3 \mathrm{~B}$ & 107.6 & $\mathrm{H} 42 \mathrm{~B}-\mathrm{C} 42-\mathrm{H} 42 \mathrm{C}$ & 109.5 \\
\hline $\mathrm{C} 3-\mathrm{C} 4-\mathrm{H} 4 \mathrm{~A}$ & 109.5 & $\mathrm{C} 38-\mathrm{C} 43-\mathrm{H} 43 \mathrm{~A}$ & 109.5 \\
\hline $\mathrm{C} 3-\mathrm{C} 4-\mathrm{H} 4 \mathrm{~B}$ & 109.5 & $\mathrm{C} 38-\mathrm{C} 43-\mathrm{H} 43 \mathrm{~B}$ & 109.5 \\
\hline $\mathrm{H} 4 \mathrm{~A}-\mathrm{C} 4-\mathrm{H} 4 \mathrm{~B}$ & 109.5 & $\mathrm{H} 43 \mathrm{~A}-\mathrm{C} 43-\mathrm{H} 43 \mathrm{~B}$ & 109.5 \\
\hline $\mathrm{C} 3-\mathrm{C} 4-\mathrm{H} 4 \mathrm{C}$ & 109.5 & $\mathrm{C} 38-\mathrm{C} 43-\mathrm{H} 43 \mathrm{C}$ & 109.5 \\
\hline $\mathrm{H} 4 \mathrm{~A}-\mathrm{C} 4-\mathrm{H} 4 \mathrm{C}$ & 109.5 & $\mathrm{H} 43 \mathrm{~A}-\mathrm{C} 43-\mathrm{H} 43 \mathrm{C}$ & 109.5 \\
\hline $\mathrm{H} 4 \mathrm{~B}-\mathrm{C} 4-\mathrm{H} 4 \mathrm{C}$ & 109.5 & $\mathrm{H} 43 \mathrm{~B}-\mathrm{C} 43-\mathrm{H} 43 \mathrm{C}$ & 109.5 \\
\hline C6-C5-Hf1 & $117.75(17)$ & $\mathrm{C} 39-\mathrm{C} 44-\mathrm{H} 44 \mathrm{~A}$ & 109.5 \\
\hline $\mathrm{C} 6-\mathrm{C} 5-\mathrm{H} 5 \mathrm{~A}$ & 107.9 & $\mathrm{C} 39-\mathrm{C} 44-\mathrm{H} 44 \mathrm{~B}$ & 109.5 \\
\hline $\mathrm{Hf} 1-\mathrm{C} 5-\mathrm{H} 5 \mathrm{~A}$ & 107.9 & $\mathrm{H} 44 \mathrm{~A}-\mathrm{C} 44-\mathrm{H} 44 \mathrm{~B}$ & 109.5 \\
\hline $\mathrm{C} 6-\mathrm{C} 5-\mathrm{H} 5 \mathrm{~B}$ & 107.9 & $\mathrm{C} 39-\mathrm{C} 44-\mathrm{H} 44 \mathrm{C}$ & 109.5 \\
\hline $\mathrm{Hf} 1-\mathrm{C} 5-\mathrm{H} 5 \mathrm{~B}$ & 107.9 & $\mathrm{H} 44 \mathrm{~A}-\mathrm{C} 44-\mathrm{H} 44 \mathrm{C}$ & 109.5 \\
\hline $\mathrm{H} 5 \mathrm{~A}-\mathrm{C} 5-\mathrm{H} 5 \mathrm{~B}$ & 107.2 & $\mathrm{H} 44 \mathrm{~B}-\mathrm{C} 44-\mathrm{H} 44 \mathrm{C}$ & 109.5 \\
\hline $\mathrm{C} 7-\mathrm{C} 6-\mathrm{C} 5$ & $112.8(2)$ & $\mathrm{C} 40-\mathrm{C} 45-\mathrm{H} 45 \mathrm{~A}$ & 109.5 \\
\hline $\mathrm{C} 7-\mathrm{C} 6-\mathrm{H} 6 \mathrm{~A}$ & 109.0 & $\mathrm{C} 40-\mathrm{C} 45-\mathrm{H} 45 \mathrm{~B}$ & 109.5 \\
\hline $\mathrm{C} 5-\mathrm{C} 6-\mathrm{H} 6 \mathrm{~A}$ & 109.0 & $\mathrm{H} 45 \mathrm{~A}-\mathrm{C} 45-\mathrm{H} 45 \mathrm{~B}$ & 109.5 \\
\hline $\mathrm{C} 7-\mathrm{C} 6-\mathrm{H} 6 \mathrm{~B}$ & 109.0 & $\mathrm{C} 40-\mathrm{C} 45-\mathrm{H} 45 \mathrm{C}$ & 109.5 \\
\hline $\mathrm{C} 5-\mathrm{C} 6-\mathrm{H} 6 \mathrm{~B}$ & 109.0 & $\mathrm{H} 45 \mathrm{~A}-\mathrm{C} 45-\mathrm{H} 45 \mathrm{C}$ & 109.5 \\
\hline $\mathrm{H} 6 \mathrm{~A}-\mathrm{C} 6-\mathrm{H} 6 \mathrm{~B}$ & 107.8 & $\mathrm{H} 45 \mathrm{~B}-\mathrm{C} 45-\mathrm{H} 45 \mathrm{C}$ & 109.5 \\
\hline $\mathrm{C} 8-\mathrm{C} 7-\mathrm{C} 6$ & $113.6(2)$ & $\mathrm{C} 41-\mathrm{C} 46-\mathrm{H} 46 \mathrm{~A}$ & 109.5 \\
\hline $\mathrm{C} 8-\mathrm{C} 7-\mathrm{H} 7 \mathrm{~A}$ & 108.8 & $\mathrm{C} 41-\mathrm{C} 46-\mathrm{H} 46 \mathrm{~B}$ & 109.5 \\
\hline $\mathrm{C} 6-\mathrm{C} 7-\mathrm{H} 7 \mathrm{~A}$ & 108.8 & $\mathrm{H} 46 \mathrm{~A}-\mathrm{C} 46-\mathrm{H} 46 \mathrm{~B}$ & 109.5 \\
\hline
\end{tabular}




\begin{tabular}{|c|c|c|c|}
\hline $\mathrm{C} 8-\mathrm{C} 7-\mathrm{H} 7 \mathrm{~B}$ & 108.8 & $\mathrm{C} 41-\mathrm{C} 46-\mathrm{H} 46 \mathrm{C}$ & 109.5 \\
\hline $\mathrm{C} 6-\mathrm{C} 7-\mathrm{H} 7 \mathrm{~B}$ & 108.8 & $\mathrm{H} 46 \mathrm{~A}-\mathrm{C} 46-\mathrm{H} 46 \mathrm{C}$ & 109.5 \\
\hline $\mathrm{H} 7 \mathrm{~A}-\mathrm{C} 7-\mathrm{H} 7 \mathrm{~B}$ & 107.7 & $\mathrm{H} 46 \mathrm{~B}-\mathrm{C} 46-\mathrm{H} 46 \mathrm{C}$ & 109.5 \\
\hline $\mathrm{C} 7-\mathrm{C} 8-\mathrm{H} 8 \mathrm{~A}$ & 109.5 & $\mathrm{C} 48-\mathrm{C} 47-\mathrm{C} 51$ & $108.1(2)$ \\
\hline $\mathrm{C} 7-\mathrm{C} 8-\mathrm{H} 8 \mathrm{~B}$ & 109.5 & $\mathrm{C} 48-\mathrm{C} 47-\mathrm{C} 52$ & $126.8(2)$ \\
\hline $\mathrm{H} 8 \mathrm{~A}-\mathrm{C} 8-\mathrm{H} 8 \mathrm{~B}$ & 109.5 & $\mathrm{C} 51-\mathrm{C} 47-\mathrm{C} 52$ & $124.3(2)$ \\
\hline $\mathrm{C} 7-\mathrm{C} 8-\mathrm{H} 8 \mathrm{C}$ & 109.5 & $\mathrm{C} 48-\mathrm{C} 47-\mathrm{Hf} 2$ & $74.54(14)$ \\
\hline $\mathrm{H} 8 \mathrm{~A}-\mathrm{C} 8-\mathrm{H} 8 \mathrm{C}$ & 109.5 & C51-C47-Hf2 & $74.62(14)$ \\
\hline $\mathrm{H} 8 \mathrm{~B}-\mathrm{C} 8-\mathrm{H} 8 \mathrm{C}$ & 109.5 & $\mathrm{C} 52-\mathrm{C} 47-\mathrm{Hf} 2$ & $125.20(18)$ \\
\hline $\mathrm{C} 13-\mathrm{C} 9-\mathrm{C} 10$ & $108.4(2)$ & $\mathrm{C} 49-\mathrm{C} 48-\mathrm{C} 47$ & $108.5(2)$ \\
\hline $\mathrm{C} 13-\mathrm{C} 9-\mathrm{C} 14$ & $123.0(3)$ & $\mathrm{C} 49-\mathrm{C} 48-\mathrm{C} 53$ & $125.1(2)$ \\
\hline $\mathrm{C} 10-\mathrm{C} 9-\mathrm{C} 14$ & $126.7(3)$ & $\mathrm{C} 47-\mathrm{C} 48-\mathrm{C} 53$ & $125.7(2)$ \\
\hline $\mathrm{C} 13-\mathrm{C} 9-\mathrm{Hfl}$ & $74.90(15)$ & $\mathrm{C} 49-\mathrm{C} 48-\mathrm{Hf} 2$ & $73.80(14)$ \\
\hline $\mathrm{C} 10-\mathrm{C} 9-\mathrm{Hfl}$ & $73.30(14)$ & $\mathrm{C} 47-\mathrm{C} 48-\mathrm{Hf} 2$ & $73.49(14)$ \\
\hline $\mathrm{C} 14-\mathrm{C} 9-\mathrm{Hf1}$ & $130.23(18)$ & $\mathrm{C} 53-\mathrm{C} 48-\mathrm{Hf} 2$ & $126.25(18)$ \\
\hline $\mathrm{C} 11-\mathrm{C} 10-\mathrm{C} 9$ & $107.3(2)$ & $\mathrm{C} 48-\mathrm{C} 49-\mathrm{C} 50$ & $107.7(3)$ \\
\hline $\mathrm{C} 11-\mathrm{C} 10-\mathrm{C} 15$ & $124.5(2)$ & $\mathrm{C} 48-\mathrm{C} 49-\mathrm{C} 54$ & $126.1(2)$ \\
\hline $\mathrm{C} 9-\mathrm{C} 10-\mathrm{C} 15$ & $127.5(3)$ & $\mathrm{C} 50-\mathrm{C} 49-\mathrm{C} 54$ & $124.9(3)$ \\
\hline $\mathrm{C} 11-\mathrm{C} 10-\mathrm{Hf} 1$ & $75.53(15)$ & $\mathrm{C} 48-\mathrm{C} 49-\mathrm{Hf} 2$ & $74.31(14)$ \\
\hline $\mathrm{C} 9-\mathrm{C} 10-\mathrm{Hf1}$ & $74.05(14)$ & C50-C49-Hf2 & $72.84(14)$ \\
\hline $\mathrm{C} 15-\mathrm{C} 10-\mathrm{Hf} 1$ & $123.85(18)$ & $\mathrm{C} 54-\mathrm{C} 49-\mathrm{Hf} 2$ & $128.65(19)$ \\
\hline $\mathrm{C} 12-\mathrm{C} 11-\mathrm{C} 10$ & $108.3(2)$ & $\mathrm{C} 51-\mathrm{C} 50-\mathrm{C} 49$ & $108.0(2)$ \\
\hline $\mathrm{C} 12-\mathrm{C} 11-\mathrm{C} 16$ & $124.7(3)$ & $\mathrm{C} 51-\mathrm{C} 50-\mathrm{C} 55$ & $127.1(3)$ \\
\hline $\mathrm{C} 10-\mathrm{C} 11-\mathrm{C} 16$ & $126.0(2)$ & $\mathrm{C} 49-\mathrm{C} 50-\mathrm{C} 55$ & $124.3(3)$ \\
\hline $\mathrm{C} 12-\mathrm{C} 11-\mathrm{Hf} 1$ & $74.36(13)$ & $\mathrm{C} 51-\mathrm{C} 50-\mathrm{Hf} 2$ & $75.26(16)$ \\
\hline $\mathrm{C} 10-\mathrm{C} 11-\mathrm{Hf} 1$ & $72.16(13)$ & $\mathrm{C} 49-\mathrm{C} 50-\mathrm{Hf} 2$ & $74.81(15)$ \\
\hline $\mathrm{C} 16-\mathrm{C} 11-\mathrm{Hf} 1$ & $128.38(19)$ & $\mathrm{C} 55-\mathrm{C} 50-\mathrm{Hf} 2$ & $123.0(2)$ \\
\hline $\mathrm{C} 11-\mathrm{C} 12-\mathrm{C} 13$ & $107.8(3)$ & $\mathrm{C} 47-\mathrm{C} 51-\mathrm{C} 50$ & $107.7(2)$ \\
\hline $\mathrm{C} 11-\mathrm{C} 12-\mathrm{C} 17$ & $124.0(3)$ & $\mathrm{C} 47-\mathrm{C} 51-\mathrm{C} 56$ & $123.7(3)$ \\
\hline $\mathrm{C} 13-\mathrm{C} 12-\mathrm{C} 17$ & $127.9(3)$ & $\mathrm{C} 50-\mathrm{C} 51-\mathrm{C} 56$ & $126.6(3)$ \\
\hline $\mathrm{C} 11-\mathrm{C} 12-\mathrm{Hf} 1$ & $73.76(13)$ & $\mathrm{C} 47-\mathrm{C} 51-\mathrm{Hf} 2$ & $73.31(14)$ \\
\hline $\mathrm{C} 13-\mathrm{C} 12-\mathrm{Hf1}$ & $73.26(14)$ & C50- C $51-\mathrm{Hf} 2$ & $72.52(16)$ \\
\hline $\mathrm{C} 17-\mathrm{C} 12-\mathrm{Hf} 1$ & $123.89(19)$ & $\mathrm{C} 56-\mathrm{C} 51-\mathrm{Hf} 2$ & $132.66(18)$ \\
\hline $\mathrm{C} 9-\mathrm{C} 13-\mathrm{C} 12$ & $108.1(2)$ & $\mathrm{C} 47-\mathrm{C} 52-\mathrm{H} 52 \mathrm{~A}$ & 109.5 \\
\hline $\mathrm{C} 9-\mathrm{C} 13-\mathrm{C} 18$ & $125.4(3)$ & $\mathrm{C} 47-\mathrm{C} 52-\mathrm{H} 52 \mathrm{~B}$ & 109.5 \\
\hline $\mathrm{C} 12-\mathrm{C} 13-\mathrm{C} 18$ & $126.1(3)$ & $\mathrm{H} 52 \mathrm{~A}-\mathrm{C} 52-\mathrm{H} 52 \mathrm{~B}$ & 109.5 \\
\hline C9-C13-Hf1 & $73.09(14)$ & $\mathrm{C} 47-\mathrm{C} 52-\mathrm{H} 52 \mathrm{C}$ & 109.5 \\
\hline $\mathrm{C} 12-\mathrm{C} 13-\mathrm{Hf1}$ & $74.66(14)$ & $\mathrm{H} 52 \mathrm{~A}-\mathrm{C} 52-\mathrm{H} 52 \mathrm{C}$ & 109.5 \\
\hline $\mathrm{C} 18-\mathrm{C} 13-\mathrm{Hf} 1$ & $124.00(18)$ & $\mathrm{H} 52 \mathrm{~B}-\mathrm{C} 52-\mathrm{H} 52 \mathrm{C}$ & 109.5 \\
\hline C9- C14-H14A & 109.5 & $\mathrm{C} 48-\mathrm{C} 53-\mathrm{H} 53 \mathrm{~A}$ & 109.5 \\
\hline $\mathrm{C} 9-\mathrm{C} 14-\mathrm{H} 14 \mathrm{~B}$ & 109.5 & $\mathrm{C} 48-\mathrm{C} 53-\mathrm{H} 53 \mathrm{~B}$ & 109.5 \\
\hline $\mathrm{H} 14 \mathrm{~A}-\mathrm{C} 14-\mathrm{H} 14 \mathrm{~B}$ & 109.5 & $\mathrm{H} 53 \mathrm{~A}-\mathrm{C} 53-\mathrm{H} 53 \mathrm{~B}$ & 109.5 \\
\hline $\mathrm{C} 9-\mathrm{C} 14-\mathrm{H} 14 \mathrm{C}$ & 109.5 & $\mathrm{C} 48-\mathrm{C} 53-\mathrm{H} 53 \mathrm{C}$ & 109.5 \\
\hline $\mathrm{H} 14 \mathrm{~A}-\mathrm{C} 14-\mathrm{H} 14 \mathrm{C}$ & 109.5 & $\mathrm{H} 53 \mathrm{~A}-\mathrm{C} 53-\mathrm{H} 53 \mathrm{C}$ & 109.5 \\
\hline $\mathrm{H} 14 \mathrm{~B}-\mathrm{C} 14-\mathrm{H} 14 \mathrm{C}$ & 109.5 & $\mathrm{H} 53 \mathrm{~B}-\mathrm{C} 53-\mathrm{H} 53 \mathrm{C}$ & 109.5 \\
\hline $\mathrm{C} 10-\mathrm{C} 15-\mathrm{H} 15 \mathrm{~A}$ & 109.5 & $\mathrm{C} 49-\mathrm{C} 54-\mathrm{H} 54 \mathrm{~A}$ & 109.5 \\
\hline $\mathrm{C} 10-\mathrm{C} 15-\mathrm{H} 15 \mathrm{~B}$ & 109.5 & $\mathrm{C} 49-\mathrm{C} 54-\mathrm{H} 54 \mathrm{~B}$ & 109.5 \\
\hline $\mathrm{H} 15 \mathrm{~A}-\mathrm{C} 15-\mathrm{H} 15 \mathrm{~B}$ & 109.5 & $\mathrm{H} 54 \mathrm{~A}-\mathrm{C} 54-\mathrm{H} 54 \mathrm{~B}$ & 109.5 \\
\hline
\end{tabular}




\begin{tabular}{|c|c|c|c|}
\hline $\mathrm{C} 10-\mathrm{C} 15-\mathrm{H} 15 \mathrm{C}$ & 109.5 & $\mathrm{C} 49-\mathrm{C} 54-\mathrm{H} 54 \mathrm{C}$ & 109.5 \\
\hline $\mathrm{H} 15 \mathrm{~A}-\mathrm{C} 15-\mathrm{H} 15 \mathrm{C}$ & 109.5 & $\mathrm{H} 54 \mathrm{~A}-\mathrm{C} 54-\mathrm{H} 54 \mathrm{C}$ & 109.5 \\
\hline $\mathrm{H} 15 \mathrm{~B}-\mathrm{C} 15-\mathrm{H} 15 \mathrm{C}$ & 109.5 & $\mathrm{H} 54 \mathrm{~B}-\mathrm{C} 54-\mathrm{H} 54 \mathrm{C}$ & 109.5 \\
\hline $\mathrm{C} 11-\mathrm{C} 16-\mathrm{H} 16 \mathrm{~A}$ & 109.5 & $\mathrm{C} 50-\mathrm{C} 55-\mathrm{H} 55 \mathrm{~A}$ & 109.5 \\
\hline $\mathrm{C} 11-\mathrm{C} 16-\mathrm{H} 16 \mathrm{~B}$ & 109.5 & $\mathrm{C} 50-\mathrm{C} 55-\mathrm{H} 55 \mathrm{~B}$ & 109.5 \\
\hline $\mathrm{H} 16 \mathrm{~A}-\mathrm{C} 16-\mathrm{H} 16 \mathrm{~B}$ & 109.5 & $\mathrm{H} 55 \mathrm{~A}-\mathrm{C} 55-\mathrm{H} 55 \mathrm{~B}$ & 109.5 \\
\hline $\mathrm{C} 11-\mathrm{C} 16-\mathrm{H} 16 \mathrm{C}$ & 109.5 & $\mathrm{C} 50-\mathrm{C} 55-\mathrm{H} 55 \mathrm{C}$ & 109.5 \\
\hline $\mathrm{H} 16 \mathrm{~A}-\mathrm{C} 16-\mathrm{H} 16 \mathrm{C}$ & 109.5 & $\mathrm{H} 55 \mathrm{~A}-\mathrm{C} 55-\mathrm{H} 55 \mathrm{C}$ & 109.5 \\
\hline $\mathrm{H} 16 \mathrm{~B}-\mathrm{C} 16-\mathrm{H} 16 \mathrm{C}$ & 109.5 & $\mathrm{H} 55 \mathrm{~B}-\mathrm{C} 55-\mathrm{H} 55 \mathrm{C}$ & 109.5 \\
\hline $\mathrm{C} 12-\mathrm{C} 17-\mathrm{H} 17 \mathrm{~A}$ & 109.5 & $\mathrm{C} 51-\mathrm{C} 56-\mathrm{H} 56 \mathrm{~A}$ & 109.5 \\
\hline $\mathrm{C} 12-\mathrm{C} 17-\mathrm{H} 17 \mathrm{~B}$ & 109.5 & $\mathrm{C} 51-\mathrm{C} 56-\mathrm{H} 56 \mathrm{~B}$ & 109.5 \\
\hline H17A-C17-H17B & 109.5 & $\mathrm{H} 56 \mathrm{~A}-\mathrm{C} 56-\mathrm{H} 56 \mathrm{~B}$ & 109.5 \\
\hline $\mathrm{C} 12-\mathrm{C} 17-\mathrm{H} 17 \mathrm{C}$ & 109.5 & $\mathrm{C} 51-\mathrm{C} 56-\mathrm{H} 56 \mathrm{C}$ & 109.5 \\
\hline $\mathrm{H} 17 \mathrm{~A}-\mathrm{C} 17-\mathrm{H} 17 \mathrm{C}$ & 109.5 & $\mathrm{H} 56 \mathrm{~A}-\mathrm{C} 56-\mathrm{H} 56 \mathrm{C}$ & 109.5 \\
\hline $\mathrm{H} 17 \mathrm{~B}-\mathrm{C} 17-\mathrm{H} 17 \mathrm{C}$ & 109.5 & $\mathrm{H} 56 \mathrm{~B}-\mathrm{C} 56-\mathrm{H} 56 \mathrm{C}$ & 109.5 \\
\hline C13-C18-H18A & 109.5 & $\mathrm{C} 1-\mathrm{Hf} 1-\mathrm{C} 5$ & $93.18(10)$ \\
\hline $\mathrm{C} 13-\mathrm{C} 18-\mathrm{H} 18 \mathrm{~B}$ & 109.5 & $\mathrm{C} 1-\mathrm{Hf} 1-\mathrm{C} 23$ & $132.49(10)$ \\
\hline $\mathrm{H} 18 \mathrm{~A}-\mathrm{C} 18-\mathrm{H} 18 \mathrm{~B}$ & 109.5 & $\mathrm{C} 5-\mathrm{Hf} 1-\mathrm{C} 23$ & $91.99(10)$ \\
\hline $\mathrm{C} 13-\mathrm{C} 18-\mathrm{H} 18 \mathrm{C}$ & 109.5 & $\mathrm{C} 1-\mathrm{Hf} 1-\mathrm{C} 10$ & $100.34(9)$ \\
\hline $\mathrm{H} 18 \mathrm{~A}-\mathrm{C} 18-\mathrm{H} 18 \mathrm{C}$ & 109.5 & $\mathrm{C} 5-\mathrm{Hf} 1-\mathrm{C} 10$ & $131.00(9)$ \\
\hline $\mathrm{H} 18 \mathrm{~B}-\mathrm{C} 18-\mathrm{H} 18 \mathrm{C}$ & 109.5 & $\mathrm{C} 23-\mathrm{Hf} 1-\mathrm{C} 10$ & $111.17(9)$ \\
\hline $\mathrm{C} 23-\mathrm{C} 19-\mathrm{C} 20$ & $107.8(2)$ & $\mathrm{C} 1-\mathrm{Hf} 1-\mathrm{C} 9$ & $130.25(9)$ \\
\hline $\mathrm{C} 23-\mathrm{C} 19-\mathrm{C} 24$ & $126.7(3)$ & $\mathrm{C} 5-\mathrm{Hf} 1-\mathrm{C} 9$ & $108.73(10)$ \\
\hline $\mathrm{C} 20-\mathrm{C} 19-\mathrm{C} 24$ & $123.6(3)$ & $\mathrm{C} 23-\mathrm{Hf} 1-\mathrm{C} 9$ & $91.87(8)$ \\
\hline $\mathrm{C} 23-\mathrm{C} 19-\mathrm{Hf} 1$ & $72.32(16)$ & $\mathrm{C} 10-\mathrm{Hf} 1-\mathrm{C} 9$ & $32.65(9)$ \\
\hline $\mathrm{C} 20-\mathrm{C} 19-\mathrm{Hf} 1$ & $74.20(14)$ & $\mathrm{C} 1-\mathrm{Hf} 1-\mathrm{C} 19$ & $120.14(10)$ \\
\hline $\mathrm{C} 24-\mathrm{C} 19-\mathrm{Hf} 1$ & $131.54(18)$ & $\mathrm{C} 5-\mathrm{Hf} 1-\mathrm{C} 19$ & $124.09(10)$ \\
\hline $\mathrm{C} 21-\mathrm{C} 20-\mathrm{C} 19$ & $108.1(2)$ & $\mathrm{C} 23-\mathrm{Hf} 1-\mathrm{C} 19$ & $32.19(9)$ \\
\hline $\mathrm{C} 21-\mathrm{C} 20-\mathrm{C} 25$ & $126.9(3)$ & $\mathrm{C} 10-\mathrm{Hf} 1-\mathrm{C} 19$ & $88.55(9)$ \\
\hline $\mathrm{C} 19-\mathrm{C} 20-\mathrm{C} 25$ & $124.1(2)$ & C9-Hf1-C19 & $83.79(8)$ \\
\hline $\mathrm{C} 21-\mathrm{C} 20-\mathrm{Hf} 1$ & $74.69(15)$ & $\mathrm{C} 1-\mathrm{Hf} 1-\mathrm{C} 13$ & $121.94(10)$ \\
\hline $\mathrm{C} 19-\mathrm{C} 20-\mathrm{Hf} 1$ & $73.65(14)$ & $\mathrm{C} 5-\mathrm{Hf} 1-\mathrm{C} 13$ & $79.26(9)$ \\
\hline $\mathrm{C} 25-\mathrm{C} 20-\mathrm{Hf} 1$ & $126.10(19)$ & $\mathrm{C} 23-\mathrm{Hf} 1-\mathrm{C} 13$ & $105.43(9)$ \\
\hline $\mathrm{C} 20-\mathrm{C} 21-\mathrm{C} 22$ & $107.9(2)$ & $\mathrm{C} 10-\mathrm{Hf} 1-\mathrm{C} 13$ & $53.63(8)$ \\
\hline $\mathrm{C} 20-\mathrm{C} 21-\mathrm{C} 26$ & $126.7(3)$ & C9-Hf1-C13 & $32.01(9)$ \\
\hline $\mathrm{C} 22-\mathrm{C} 21-\mathrm{C} 26$ & $124.5(2)$ & $\mathrm{C} 19-\mathrm{Hf} 1-\mathrm{C} 13$ & $110.79(9)$ \\
\hline $\mathrm{C} 20-\mathrm{C} 21-\mathrm{Hf} 1$ & $73.37(15)$ & $\mathrm{C} 1-\mathrm{Hf} 1-\mathrm{C} 22$ & $104.10(9)$ \\
\hline $\mathrm{C} 22-\mathrm{C} 21-\mathrm{Hf} 1$ & $73.31(14)$ & $\mathrm{C} 5-\mathrm{Hf} 1-\mathrm{C} 22$ & $77.24(9)$ \\
\hline $\mathrm{C} 26-\mathrm{C} 21-\mathrm{Hf} 1$ & $127.43(17)$ & $\mathrm{C} 23-\mathrm{Hf} 1-\mathrm{C} 22$ & $32.35(8)$ \\
\hline $\mathrm{C} 23-\mathrm{C} 22-\mathrm{C} 21$ & $107.7(2)$ & $\mathrm{C} 10-\mathrm{Hf} 1-\mathrm{C} 22$ & $141.24(9)$ \\
\hline $\mathrm{C} 23-\mathrm{C} 22-\mathrm{C} 27$ & $126.3(3)$ & C9-Hf1-C22 & $123.75(8)$ \\
\hline $\mathrm{C} 21-\mathrm{C} 22-\mathrm{C} 27$ & $125.0(2)$ & $\mathrm{C} 19-\mathrm{Hf} 1-\mathrm{C} 22$ & $53.15(9)$ \\
\hline $\mathrm{C} 23-\mathrm{C} 22-\mathrm{Hf} 1$ & 71.99 (14) & $\mathrm{C} 13-\mathrm{Hf} 1-\mathrm{C} 22$ & $128.94(8)$ \\
\hline $\mathrm{C} 21-\mathrm{C} 22-\mathrm{Hf} 1$ & $74.66(14)$ & $\mathrm{C} 1-\mathrm{Hf} 1-\mathrm{C} 20$ & $88.24(9)$ \\
\hline $\mathrm{C} 27-\mathrm{C} 22-\mathrm{Hf} 1$ & $128.22(19)$ & $\mathrm{C} 5-\mathrm{Hf} 1-\mathrm{C} 20$ & $128.82(9)$ \\
\hline $\mathrm{C} 19-\mathrm{C} 23-\mathrm{C} 22$ & $108.5(2)$ & $\mathrm{C} 23-\mathrm{Hf} 1-\mathrm{C} 20$ & $53.40(9)$ \\
\hline $\mathrm{C} 19-\mathrm{C} 23-\mathrm{C} 28$ & $127.0(3)$ & $\mathrm{C} 10-\mathrm{Hf} 1-\mathrm{C} 20$ & $98.70(8)$ \\
\hline $\mathrm{C} 22-\mathrm{C} 23-\mathrm{C} 28$ & $124.1(3)$ & C9-Hf1-C20 & $108.75(8)$ \\
\hline
\end{tabular}




\begin{tabular}{|c|c|c|c|}
\hline $\mathrm{C} 19-\mathrm{C} 23-\mathrm{Hf} 1$ & $75.49(16)$ & $\mathrm{C} 19-\mathrm{Hf} 1-\mathrm{C} 20$ & $32.15(8)$ \\
\hline $\mathrm{C} 22-\mathrm{C} 23-\mathrm{Hf} 1$ & $75.66(14)$ & $\mathrm{C} 13-\mathrm{Hf} 1-\mathrm{C} 20$ & $139.86(9)$ \\
\hline $\mathrm{C} 28-\mathrm{C} 23-\mathrm{Hf} 1$ & 121.07 (19) & $\mathrm{C} 22-\mathrm{Hf} 1-\mathrm{C} 20$ & $53.09(9)$ \\
\hline $\mathrm{C} 19-\mathrm{C} 24-\mathrm{H} 24 \mathrm{~A}$ & 109.5 & $\mathrm{C} 1-\mathrm{Hf} 1-\mathrm{C} 11$ & $77.56(10)$ \\
\hline $\mathrm{C} 19-\mathrm{C} 24-\mathrm{H} 24 \mathrm{~B}$ & 109.5 & $\mathrm{C} 5-\mathrm{Hf} 1-\mathrm{C} 11$ & $109.83(9)$ \\
\hline $\mathrm{H} 24 \mathrm{~A}-\mathrm{C} 24-\mathrm{H} 24 \mathrm{~B}$ & 109.5 & $\mathrm{C} 23-\mathrm{Hf} 1-\mathrm{C} 11$ & $142.83(10)$ \\
\hline $\mathrm{C} 19-\mathrm{C} 24-\mathrm{H} 24 \mathrm{C}$ & 109.5 & $\mathrm{C} 10-\mathrm{Hf} 1-\mathrm{C} 11$ & $32.31(10)$ \\
\hline $\mathrm{H} 24 \mathrm{~A}-\mathrm{C} 24-\mathrm{H} 24 \mathrm{C}$ & 109.5 & C9-Hf1-C11 & $53.26(9)$ \\
\hline $\mathrm{H} 24 \mathrm{~B}-\mathrm{C} 24-\mathrm{H} 24 \mathrm{C}$ & 109.5 & C19-Hf1-C11 & $119.82(9)$ \\
\hline $\mathrm{C} 20-\mathrm{C} 25-\mathrm{H} 25 \mathrm{~A}$ & 109.5 & $\mathrm{C} 13-\mathrm{Hf} 1-\mathrm{C} 11$ & $53.02(8)$ \\
\hline $\mathrm{C} 20-\mathrm{C} 25-\mathrm{H} 25 \mathrm{~B}$ & 109.5 & $\mathrm{C} 22-\mathrm{Hf} 1-\mathrm{C} 11$ & $172.75(9)$ \\
\hline $\mathrm{H} 25 \mathrm{~A}-\mathrm{C} 25-\mathrm{H} 25 \mathrm{~B}$ & 109.5 & $\mathrm{C} 20-\mathrm{Hf} 1-\mathrm{C} 11$ & $120.31(8)$ \\
\hline $\mathrm{C} 20-\mathrm{C} 25-\mathrm{H} 25 \mathrm{C}$ & 109.5 & $\mathrm{C} 1-\mathrm{Hf} 1-\mathrm{C} 12$ & $89.86(9)$ \\
\hline $\mathrm{H} 25 \mathrm{~A}-\mathrm{C} 25-\mathrm{H} 25 \mathrm{C}$ & 109.5 & $\mathrm{C} 5-\mathrm{Hf} 1-\mathrm{C} 12$ & $79.99(9)$ \\
\hline $\mathrm{H} 25 \mathrm{~B}-\mathrm{C} 25-\mathrm{H} 25 \mathrm{C}$ & 109.5 & $\mathrm{C} 23-\mathrm{Hf} 1-\mathrm{C} 12$ & $137.47(9)$ \\
\hline $\mathrm{C} 21-\mathrm{C} 26-\mathrm{H} 26 \mathrm{~A}$ & 109.5 & $\mathrm{C} 10-\mathrm{Hf} 1-\mathrm{C} 12$ & $53.46(9)$ \\
\hline $\mathrm{C} 21-\mathrm{C} 26-\mathrm{H} 26 \mathrm{~B}$ & 109.5 & C9-Hf1-C12 & $53.12(8)$ \\
\hline $\mathrm{H} 26 \mathrm{~A}-\mathrm{C} 26-\mathrm{H} 26 \mathrm{~B}$ & 109.5 & $\mathrm{C} 19-\mathrm{Hf} 1-\mathrm{C} 12$ & $136.66(9)$ \\
\hline $\mathrm{C} 21-\mathrm{C} 26-\mathrm{H} 26 \mathrm{C}$ & 109.5 & $\mathrm{C} 13-\mathrm{Hf} 1-\mathrm{C} 12$ & $32.09(9)$ \\
\hline $\mathrm{H} 26 \mathrm{~A}-\mathrm{C} 26-\mathrm{H} 26 \mathrm{C}$ & 109.5 & $\mathrm{C} 22-\mathrm{Hf} 1-\mathrm{C} 12$ & $153.81(8)$ \\
\hline $\mathrm{H} 26 \mathrm{~B}-\mathrm{C} 26-\mathrm{H} 26 \mathrm{C}$ & 109.5 & $\mathrm{C} 20-\mathrm{Hf} 1-\mathrm{C} 12$ & $151.19(8)$ \\
\hline $\mathrm{C} 22-\mathrm{C} 27-\mathrm{H} 27 \mathrm{~A}$ & 109.5 & $\mathrm{C} 11-\mathrm{Hf} 1-\mathrm{C} 12$ & $31.88(8)$ \\
\hline $\mathrm{C} 22-\mathrm{C} 27-\mathrm{H} 27 \mathrm{~B}$ & 109.5 & $\mathrm{C} 1-\mathrm{Hf} 1-\mathrm{C} 21$ & $79.19(9)$ \\
\hline $\mathrm{H} 27 \mathrm{~A}-\mathrm{C} 27-\mathrm{H} 27 \mathrm{~B}$ & 109.5 & $\mathrm{C} 5-\mathrm{Hf} 1-\mathrm{C} 21$ & $98.39(9)$ \\
\hline $\mathrm{C} 22-\mathrm{C} 27-\mathrm{H} 27 \mathrm{C}$ & 109.5 & $\mathrm{C} 23-\mathrm{Hf} 1-\mathrm{C} 21$ & $53.34(8)$ \\
\hline $\mathrm{H} 27 \mathrm{~A}-\mathrm{C} 27-\mathrm{H} 27 \mathrm{C}$ & 109.5 & $\mathrm{C} 10-\mathrm{Hf} 1-\mathrm{C} 21$ & $130.28(9)$ \\
\hline $\mathrm{H} 27 \mathrm{~B}-\mathrm{C} 27-\mathrm{H} 27 \mathrm{C}$ & 109.5 & C9-Hf1-C21 & $136.78(8)$ \\
\hline $\mathrm{C} 23-\mathrm{C} 28-\mathrm{H} 28 \mathrm{~A}$ & 109.5 & $\mathrm{C} 19-\mathrm{Hf} 1-\mathrm{C} 21$ & $53.02(8)$ \\
\hline $\mathrm{C} 23-\mathrm{C} 28-\mathrm{H} 28 \mathrm{~B}$ & 109.5 & $\mathrm{C} 13-\mathrm{Hf} 1-\mathrm{C} 21$ & $158.72(9)$ \\
\hline $\mathrm{H} 28 \mathrm{~A}-\mathrm{C} 28-\mathrm{H} 28 \mathrm{~B}$ & 109.5 & $\mathrm{C} 22-\mathrm{Hf} 1-\mathrm{C} 21$ & $32.03(8)$ \\
\hline $\mathrm{C} 23-\mathrm{C} 28-\mathrm{H} 28 \mathrm{C}$ & 109.5 & $\mathrm{C} 20-\mathrm{Hf} 1-\mathrm{C} 21$ & $31.93(8)$ \\
\hline $\mathrm{H} 28 \mathrm{~A}-\mathrm{C} 28-\mathrm{H} 28 \mathrm{C}$ & 109.5 & $\mathrm{C} 11-\mathrm{Hf} 1-\mathrm{C} 21$ & $144.09(8)$ \\
\hline $\mathrm{H} 28 \mathrm{~B}-\mathrm{C} 28-\mathrm{H} 28 \mathrm{C}$ & 109.5 & $\mathrm{C} 12-\mathrm{Hf} 1-\mathrm{C} 21$ & $168.85(8)$ \\
\hline $\mathrm{C} 30-\mathrm{C} 29-\mathrm{Hf} 2$ & $131.33(18)$ & $\mathrm{C} 29-\mathrm{Hf} 2-\mathrm{C} 33$ & $91.61(9)$ \\
\hline $\mathrm{C} 30-\mathrm{C} 29-\mathrm{H} 29 \mathrm{~A}$ & 104.4 & $\mathrm{C} 29-\mathrm{Hf} 2-\mathrm{C} 37$ & $98.01(10)$ \\
\hline $\mathrm{Hf} 2-\mathrm{C} 29-\mathrm{H} 29 \mathrm{~A}$ & 104.4 & $\mathrm{C} 33-\mathrm{Hf} 2-\mathrm{C} 37$ & $131.48(9)$ \\
\hline $\mathrm{C} 30-\mathrm{C} 29-\mathrm{H} 29 \mathrm{~B}$ & 104.4 & $\mathrm{C} 29-\mathrm{Hf} 2-\mathrm{C} 41$ & $128.90(9)$ \\
\hline Hf2-C29-H29B & 104.4 & $\mathrm{C} 33-\mathrm{Hf} 2-\mathrm{C} 41$ & $112.16(10)$ \\
\hline $\mathrm{H} 29 \mathrm{~A}-\mathrm{C} 29-\mathrm{H} 29 \mathrm{~B}$ & 105.6 & C37-Hf2-C41 & $32.66(9)$ \\
\hline $\mathrm{C} 31-\mathrm{C} 30-\mathrm{C} 29$ & $112.4(2)$ & $\mathrm{C} 29-\mathrm{Hf} 2-\mathrm{C} 50$ & $133.69(9)$ \\
\hline $\mathrm{C} 31-\mathrm{C} 30-\mathrm{H} 30 \mathrm{~A}$ & 109.1 & $\mathrm{C} 33-\mathrm{Hf} 2-\mathrm{C} 50$ & $94.00(10)$ \\
\hline $\mathrm{C} 29-\mathrm{C} 30-\mathrm{H} 30 \mathrm{~A}$ & 109.1 & $\mathrm{C} 37-\mathrm{Hf} 2-\mathrm{C} 50$ & $111.71(10)$ \\
\hline $\mathrm{C} 31-\mathrm{C} 30-\mathrm{H} 30 \mathrm{~B}$ & 109.1 & $\mathrm{C} 41-\mathrm{Hf} 2-\mathrm{C} 50$ & $90.70(9)$ \\
\hline $\mathrm{C} 29-\mathrm{C} 30-\mathrm{H} 30 \mathrm{~B}$ & 109.1 & $\mathrm{C} 29-\mathrm{Hf} 2-\mathrm{C} 40$ & $123.36(10)$ \\
\hline $\mathrm{H} 30 \mathrm{~A}-\mathrm{C} 30-\mathrm{H} 30 \mathrm{~B}$ & 107.9 & $\mathrm{C} 33-\mathrm{Hf} 2-\mathrm{C} 40$ & $81.39(9)$ \\
\hline $\mathrm{C} 32-\mathrm{C} 31-\mathrm{C} 30$ & $115.2(3)$ & C37-Hf2-C40 & $53.78(8)$ \\
\hline $\mathrm{C} 32-\mathrm{C} 31-\mathrm{H} 31 \mathrm{~A}$ & 108.5 & $\mathrm{C} 41-\mathrm{Hf} 2-\mathrm{C} 40$ & $32.32(9)$ \\
\hline $\mathrm{C} 30-\mathrm{C} 31-\mathrm{H} 31 \mathrm{~A}$ & 108.5 & $\mathrm{C} 50-\mathrm{Hf} 2-\mathrm{C} 40$ & $102.93(9)$ \\
\hline
\end{tabular}




\begin{tabular}{|c|c|c|c|}
\hline $\mathrm{C} 32-\mathrm{C} 31-\mathrm{H} 31 \mathrm{~B}$ & 108.5 & $\mathrm{C} 29-\mathrm{Hf} 2-\mathrm{C} 47$ & $87.98(9)$ \\
\hline $\mathrm{C} 30-\mathrm{C} 31-\mathrm{H} 31 \mathrm{~B}$ & 108.5 & $\mathrm{C} 33-\mathrm{Hf} 2-\mathrm{C} 47$ & $127.94(9)$ \\
\hline $\mathrm{H} 31 \mathrm{~A}-\mathrm{C} 31-\mathrm{H} 31 \mathrm{~B}$ & 107.5 & C37-Hf2-C47 & $99.97(9)$ \\
\hline C31-C32-H32A & 109.5 & $\mathrm{C} 41-\mathrm{Hf} 2-\mathrm{C} 47$ & $107.96(9)$ \\
\hline $\mathrm{C} 31-\mathrm{C} 32-\mathrm{H} 32 \mathrm{~B}$ & 109.5 & $\mathrm{C} 50-\mathrm{Hf} 2-\mathrm{C} 47$ & $53.41(9)$ \\
\hline $\mathrm{H} 32 \mathrm{~A}-\mathrm{C} 32-\mathrm{H} 32 \mathrm{~B}$ & 109.5 & $\mathrm{C} 40-\mathrm{Hf} 2-\mathrm{C} 47$ & $138.75(9)$ \\
\hline $\mathrm{C} 31-\mathrm{C} 32-\mathrm{H} 32 \mathrm{C}$ & 109.5 & $\mathrm{C} 29-\mathrm{Hf} 2-\mathrm{C} 49$ & $106.03(9)$ \\
\hline $\mathrm{H} 32 \mathrm{~A}-\mathrm{C} 32-\mathrm{H} 32 \mathrm{C}$ & 109.5 & $\mathrm{C} 33-\mathrm{Hf} 2-\mathrm{C} 49$ & $77.37(9)$ \\
\hline $\mathrm{H} 32 \mathrm{~B}-\mathrm{C} 32-\mathrm{H} 32 \mathrm{C}$ & 109.5 & C37-Hf2-C49 & $142.08(10)$ \\
\hline $\mathrm{C} 34-\mathrm{C} 33-\mathrm{Hf} 2$ & $117.53(17)$ & $\mathrm{C} 41-\mathrm{Hf} 2-\mathrm{C} 49$ & $122.59(9)$ \\
\hline $\mathrm{C} 34-\mathrm{C} 33-\mathrm{H} 33 \mathrm{~A}$ & 107.9 & $\mathrm{C} 50-\mathrm{Hf} 2-\mathrm{C} 49$ & $32.35(8)$ \\
\hline $\mathrm{Hf} 2-\mathrm{C} 33-\mathrm{H} 33 \mathrm{~A}$ & 107.9 & $\mathrm{C} 40-\mathrm{Hf} 2-\mathrm{C} 49$ & $126.36(9)$ \\
\hline $\mathrm{C} 34-\mathrm{C} 33-\mathrm{H} 33 \mathrm{~B}$ & 107.9 & $\mathrm{C} 47-\mathrm{Hf} 2-\mathrm{C} 49$ & $53.13(9)$ \\
\hline Hf2-C33-H33B & 107.9 & $\mathrm{C} 29-\mathrm{Hf} 2-\mathrm{C} 48$ & $80.52(9)$ \\
\hline $\mathrm{H} 33 \mathrm{~A}-\mathrm{C} 33-\mathrm{H} 33 \mathrm{~B}$ & 107.2 & $\mathrm{C} 33-\mathrm{Hf} 2-\mathrm{C} 48$ & $96.82(9)$ \\
\hline $\mathrm{C} 35-\mathrm{C} 34-\mathrm{C} 33$ & $115.5(2)$ & C37-Hf2-C48 & $131.63(8)$ \\
\hline $\mathrm{C} 35-\mathrm{C} 34-\mathrm{H} 34 \mathrm{~A}$ & 108.4 & $\mathrm{C} 41-\mathrm{Hf} 2-\mathrm{C} 48$ & $135.67(9)$ \\
\hline $\mathrm{C} 33-\mathrm{C} 34-\mathrm{H} 34 \mathrm{~A}$ & 108.4 & $\mathrm{C} 50-\mathrm{Hf} 2-\mathrm{C} 48$ & $53.17(9)$ \\
\hline C $35-\mathrm{C} 34-\mathrm{H} 34 \mathrm{~B}$ & 108.4 & $\mathrm{C} 40-\mathrm{Hf} 2-\mathrm{C} 48$ & $156.01(9)$ \\
\hline $\mathrm{C} 33-\mathrm{C} 34-\mathrm{H} 34 \mathrm{~B}$ & 108.4 & $\mathrm{C} 47-\mathrm{Hf} 2-\mathrm{C} 48$ & $31.96(8)$ \\
\hline $\mathrm{H} 34 \mathrm{~A}-\mathrm{C} 34-\mathrm{H} 34 \mathrm{~B}$ & 107.5 & $\mathrm{C} 49-\mathrm{Hf} 2-\mathrm{C} 48$ & $31.89(8)$ \\
\hline $\mathrm{C} 36-\mathrm{C} 35-\mathrm{C} 34$ & $113.2(3)$ & $\mathrm{C} 29-\mathrm{Hf} 2-\mathrm{C} 51$ & $119.57(9)$ \\
\hline $\mathrm{C} 36-\mathrm{C} 35-\mathrm{H} 35 \mathrm{~A}$ & 108.9 & $\mathrm{C} 33-\mathrm{Hf} 2-\mathrm{C} 51$ & $125.89(10)$ \\
\hline $\mathrm{C} 34-\mathrm{C} 35-\mathrm{H} 35 \mathrm{~A}$ & 108.9 & $\mathrm{C} 37-\mathrm{Hf} 2-\mathrm{C} 51$ & $89.54(9)$ \\
\hline $\mathrm{C} 36-\mathrm{C} 35-\mathrm{H} 35 \mathrm{~B}$ & 108.9 & $\mathrm{C} 41-\mathrm{Hf} 2-\mathrm{C} 51$ & $82.81(8)$ \\
\hline C $34-\mathrm{C} 35-\mathrm{H} 35 \mathrm{~B}$ & 108.9 & $\mathrm{C} 50-\mathrm{Hf} 2-\mathrm{C} 51$ & $32.22(9)$ \\
\hline $\mathrm{H} 35 \mathrm{~A}-\mathrm{C} 35-\mathrm{H} 35 \mathrm{~B}$ & 107.8 & $\mathrm{C} 40-\mathrm{Hf} 2-\mathrm{C} 51$ & $109.15(9)$ \\
\hline $\mathrm{C} 35-\mathrm{C} 36-\mathrm{H} 36 \mathrm{~A}$ & 109.5 & $\mathrm{C} 47-\mathrm{Hf} 2-\mathrm{C} 51$ & $32.07(8)$ \\
\hline $\mathrm{C} 35-\mathrm{C} 36-\mathrm{H} 36 \mathrm{~B}$ & 109.5 & $\mathrm{C} 49-\mathrm{Hf} 2-\mathrm{C} 51$ & $53.15(9)$ \\
\hline $\mathrm{H} 36 \mathrm{~A}-\mathrm{C} 36-\mathrm{H} 36 \mathrm{~B}$ & 109.5 & $\mathrm{C} 48-\mathrm{Hf} 2-\mathrm{C} 51$ & $52.86(9)$ \\
\hline $\mathrm{C} 35-\mathrm{C} 36-\mathrm{H} 36 \mathrm{C}$ & 109.5 & $\mathrm{C} 29-\mathrm{Hf} 2-\mathrm{C} 39$ & $91.27(9)$ \\
\hline $\mathrm{H} 36 \mathrm{~A}-\mathrm{C} 36-\mathrm{H} 36 \mathrm{C}$ & 109.5 & $\mathrm{C} 33-\mathrm{Hf} 2-\mathrm{C} 39$ & $79.24(9)$ \\
\hline $\mathrm{H} 36 \mathrm{~B}-\mathrm{C} 36-\mathrm{H} 36 \mathrm{C}$ & 109.5 & $\mathrm{C} 37-\mathrm{Hf} 2-\mathrm{C} 39$ & $53.24(9)$ \\
\hline $\mathrm{C} 38-\mathrm{C} 37-\mathrm{C} 41$ & $107.8(2)$ & $\mathrm{C} 41-\mathrm{Hf} 2-\mathrm{C} 39$ & $53.20(8)$ \\
\hline $\mathrm{C} 38-\mathrm{C} 37-\mathrm{C} 42$ & $124.8(3)$ & $\mathrm{C} 50-\mathrm{H} 2-\mathrm{C} 39$ & $134.93(9)$ \\
\hline $\mathrm{C} 41-\mathrm{C} 37-\mathrm{C} 42$ & $127.0(3)$ & $\mathrm{C} 40-\mathrm{Hf} 2-\mathrm{C} 39$ & $32.12(9)$ \\
\hline $\mathrm{C} 38-\mathrm{C} 37-\mathrm{Hf} 2$ & $76.13(15)$ & $\mathrm{C} 47-\mathrm{Hf} 2-\mathrm{C} 39$ & $152.81(9)$ \\
\hline C41-C37-Hf2 & $73.82(14)$ & $\mathrm{C} 49-\mathrm{Hf} 2-\mathrm{C} 39$ & $151.13(9)$ \\
\hline $\mathrm{C} 42-\mathrm{C} 37-\mathrm{Hf} 2$ & $122.37(18)$ & $\mathrm{C} 48-\mathrm{H} 22-\mathrm{C} 39$ & $170.84(9)$ \\
\hline $\mathrm{C} 39-\mathrm{C} 38-\mathrm{C} 37$ & $107.9(2)$ & $\mathrm{C} 51-\mathrm{Hf} 2-\mathrm{C} 39$ & $135.97(8)$ \\
\hline $\mathrm{C} 39-\mathrm{C} 38-\mathrm{C} 43$ & $123.9(3)$ & $\mathrm{C} 29-\mathrm{Hf} 2-\mathrm{C} 38$ & $76.90(9)$ \\
\hline $\mathrm{C} 37-\mathrm{C} 38-\mathrm{C} 43$ & $127.0(3)$ & $\mathrm{C} 33-\mathrm{Hf} 2-\mathrm{C} 38$ & $107.64(9)$ \\
\hline $\mathrm{C} 39-\mathrm{C} 38-\mathrm{Hf} 2$ & 74.07 (15) & $\mathrm{C} 37-\mathrm{Hf} 2-\mathrm{C} 38$ & $32.26(10)$ \\
\hline C37-C38-Hf2 & $71.61(13)$ & $\mathrm{C} 41-\mathrm{Hf} 2-\mathrm{C} 38$ & $53.35(9)$ \\
\hline $\mathrm{C} 43-\mathrm{C} 38-\mathrm{Hf} 2$ & $129.46(18)$ & $\mathrm{C} 50-\mathrm{Hf} 2-\mathrm{C} 38$ & $142.73(9)$ \\
\hline $\mathrm{C} 38-\mathrm{C} 39-\mathrm{C} 40$ & $108.2(2)$ & $\mathrm{C} 40-\mathrm{Hf} 2-\mathrm{C} 38$ & $53.11(9)$ \\
\hline $\mathrm{C} 38-\mathrm{C} 39-\mathrm{C} 44$ & $123.9(3)$ & $\mathrm{C} 47-\mathrm{Hf} 2-\mathrm{C} 38$ & $122.76(8)$ \\
\hline $\mathrm{C} 40-\mathrm{C} 39-\mathrm{C} 44$ & $127.4(3)$ & $\mathrm{C} 49-\mathrm{H} 2-\mathrm{C} 38$ & $174.29(9)$ \\
\hline
\end{tabular}


supporting information

C38-C39-Hf2
C40-C39-Hf2
C44-C39-Hf2

$74.25(14)$

$72.30(15)$

C48-Hf2-C38

C51-Hf2-C38

$146.89(8)$

$125.08(19)$

C39-Hf2-C38

$121.16(9)$

$31.68(9)$ 